\title{
EVIDENCE FOR THE INTERMEDIATE BROAD-LINE REGION OF REVERBERATION-MAPPED ACTIVE GALACTIC NUCLEUS PG 0052+251
}

\author{
Xue-Guang Zhang ${ }^{1,2}$ \\ ${ }^{1}$ Purple Mountain Observatory, Chinese Academy of Sciences, 2 Beijing Xi Lu, Nanjing, Jiangsu 210008, China; xgzhang@ pmo.ac.cn \\ ${ }^{2}$ Department of Physics and Astronomy, Texas A\&M University, College Station, TX 77843-4242, USA \\ Received 2011 July 4; accepted 2011 August 11; published 2011 October 24
}

\begin{abstract}
We study the properties of the broad-line region (BLR) of a well-known reverberation-mapped active galactic nucleus (AGN) in order to find reliable evidence for the intermediate BLR. We first check properties of the mapped AGN collected from the literature in the plane of $\sigma_{\mathrm{H} \beta}^{2} / \sigma_{\mathrm{H} \alpha}^{2}$ versus $R_{\mathrm{BLR}}^{\mathrm{H} \alpha} / R_{\mathrm{BLR}}^{\mathrm{H} \beta}$. Commonly, virial black hole masses based on observed broad $\mathrm{H} \alpha$ and $\mathrm{H} \beta$ should be coincidental. However, among the mapped objects, PG 0052 and NGC 4253 are two apparent outliers in the plane of $\sigma_{\mathrm{H} \beta}^{2} / \sigma_{\mathrm{H} \alpha}^{2}$ versus $R_{\mathrm{BLR}}^{\mathrm{H} \alpha} / R_{\mathrm{BLR}}^{\mathrm{H} \beta}$, which indicates that BLRs of PG 0052 and NGC 4253 have some special characters. Based on the 55 public spectra of PG 0052, the BLR of PG 0052 has been carefully studied in detail. We find that the line width ratio of the total observed broad $\mathrm{H} \alpha$ to the total observed broad $\mathrm{H} \beta$ is $\sim 0.7$, which is much smaller than the theoretical/observational value of $\sim 0.9$. Furthermore, the flux ratio of the total broad $\mathrm{H} \alpha$ to the total broad $\mathrm{H} \beta$ is about 6.8 (Balmer decrement), which is not a reasonable value for the blue quasar PG 0052+251. Moreover, properties of line cores based on the principal component analysis technique confirm that there is one inner broad component and one seriously obscured intermediate broad component in the BLR of PG 0052. If the seriously obscured intermediate BLR was accepted, properties of PG 0052 in the plane of $\sigma_{\mathrm{H} \beta}^{2} / \sigma_{\mathrm{H} \alpha}^{2}$ versus $R_{\mathrm{BLR}}^{\mathrm{H} \alpha} / R_{\mathrm{BLR}}^{\mathrm{H} \beta}$ could be reproduced, which indicates that the intermediate BLR actually is appropriate for the mapped quasar PG 0052+251. Finally, the large distance between the inner and the intermediate components of the BLR based on the results of the cross-correlation function rejects the possibility that the intermediate component is probably an extended part of the inner component of the BLR.
\end{abstract}

Key words: galaxies: active - galaxies: individual (PG 0052+251) - galaxies: nuclei - quasars: emission lines

\section{INTRODUCTION}

Through strong, broad emission lines coming from the broadline regions (BLRs) of active galactic nuclei (AGNs), the properties of the BLR of AGNs, which cannot be directly resolved by current observational techniques and instruments, have become better understood (see the reviews of Sulentic et al. 2000; Gaskell 2010; Krause et al. 2011; Denney et al. 2010; Netzer \& Marziani 2010; Sluse et al. 2011; Pancoast et al. 2011, etc., and references therein). Based on properties of the BLR of AGNs (especially virialized emission line clouds in BLR; Gaskell 1988; Wandel et al. 1999; Peterson \& Wandel 1999; Osterbrock \& Mathews 1986; Denney et al. 2009b; Gaskell 2009), the most convenient method for estimating virial black hole $(\mathrm{BH})$ masses of broad line AGNs is proposed (Vestergaard 2002; Onken et al. 2004; Peterson et al. 2004; Peterson 2010; Peterson \& Bentz 2006; Collin et al. 2006; Kelly \& Bechtold 2007):

$$
M_{\mathrm{BH}} \propto V^{2} \times R_{\mathrm{BLR}},
$$

where $V$ represents the probable rotating velocity of emission line cloude in BLR (line width of the broad emission line) and $R_{\mathrm{BLR}}$ denotes the distance between the BLR and central $\mathrm{BH}$ of AGNs (size of BLR), which can be measured by the time lag between broad line emission and continuum emission from long-period observed spectra (Kaspi et al. 2000; Peterson et al. 2004; Bentz et al. 2006, 2009; Denney et al. 2010) based on the reverberation-mapping technique (Blandford \& Mckee 1982; Peterson 1993). So far there are more than 40 nearby broad line objects for which $R_{\mathrm{BLR}}$ and virial $\mathrm{BH}$ masses have been determined (Peterson et al. 2004; Denney et al. 2010; Kaspi et al. 2005; Bentz et al. 2009, 2010b; Barth et al. 2011). One empirical relation has been found for $R_{\mathrm{BLR}}, R_{\mathrm{BLR}} \propto L_{5100}^{\sim 0.5} \propto L_{\text {line }}^{\sim 0.5}$, where $L_{5100}$ means an AGN continuum luminosity at $5100 \mathrm{~A}$ and $L_{\text {line }}$ represents broad line luminosity (Kaspi et al. 2005; Denney et al. 2010; Bentz et al. 2009; Wang \& Zhang 2003; Greene et al. 2010), and virial BH masses can be simply and conveniently estimated from single epoch spectra of broad line AGNs by line width and continuum luminosity (or broad line luminosity; Vestergaard 2002; McLure \& Dunlop 2004; Sulentic et al. 2000; Netzer \& Marziani 2010; Sulentic et al. 2006, 2007; Shen et al. 2008; Rafiee \& Hall 2011; Netzer \& Marziani 2010; Kelly \& Bechtold 2007; Greene \& Ho 2005, Denney et al. 2009a):

$$
M_{\mathrm{BH}} \propto V^{2} \times L_{5100}^{\sim 0.5} \propto V^{2} \times L_{\text {line }}^{\sim 0.5} .
$$

It is clear from the single epoch spectra of broad-line AGNs that virial BH masses based on different broad emission lines should be coincidental. However, some conflicting results have been reported. Kelly \& Bechtold (2007) have found that virial BH masses based on broad lines in UV and optical bands are coincidental as well (some simple and more recent results can also be found in Rafiee \& Hall 2011). However, Sulentic et al. (2007) have shown that UV broad-line C IV $\lambda 1545$ is a poor virial estimator. Recently, Assef et al. (2010) have found the opposite that virial $\mathrm{BH}$ mass estimates based on C IV $\lambda 1545$ are consistent with those based on Balmer lines. Different virial BH masses based on different broad emission lines probably provide some kind of deep information on the structure of the BLR of AGNs. In this paper, studying the properties of virial $\mathrm{BH}$ masses on different broad emission lines for reverberation-mapped AGNs is our main objective. In order to find more reliable results, parameters based on a reverberation-mapped AGN rather than an AGN with a single epoch spectra should be first considered. 
For recently reported reverberation-mapped AGNs, the sizes of BLRs are based on long-period varied broad-line emission lines in an optical band, especially Balmer lines. Thus, in this paper, we primarily consider the properties of Balmer lines $(\mathrm{H} \alpha$ and $\mathrm{H} \beta$ ) of the reverberation-mapped AGN.

Besides the reverberation-mapping technique and the virialization method for virial $\mathrm{BH}$ masses, there are some references that study properties of emission line regions for broad optical Balmer lines. Korista \& Goad (2004) showed that after considering luminosity-dependent responsivities, the theoretically estimated size of the BLR based on $\mathrm{H} \alpha$ should be $20 \%$ larger than the size of the BLR based on $\mathrm{H} \beta$, and the corresponding line width of $\mathrm{H} \beta$ should be larger than that of $\mathrm{H} \alpha$. The theoretical results can be confirmed by observational results shown in Bentz et al. (2010b; size of the BLR on $\mathrm{H} \alpha$ is larger than the size of the BLR on $\mathrm{H} \beta$ ) for mapped objects and in Greene \& Ho (2005) (line width of broad $\mathrm{H} \beta$ is larger than the line width of broad $\mathrm{H} \alpha$ ) for pure quasars. Furthermore, a two-component model of the BLR has been proposed (Popovic 2007; Bon et al. 2009; Zhu et al. 2009; Hu et al. 2008): one inner region for broad wings and one intermediate region for cores, in order to explain complex broad lines of AGNs. Although estimated (or measured) parameters (line width and size of the BLR) of $\mathrm{H} \alpha$ and $\mathrm{H} \beta$ are different to some extent, estimated virial $\mathrm{BH}$ masses based on $\mathrm{H} \alpha$ and $\mathrm{H} \beta$ should be coincidental, such as the confirmed results shown in Greene \& Ho (2005) for pure quasars.

It is interesting to check the virial $\mathrm{BH}$ masses based on $\mathrm{H} \alpha$ and $\mathrm{H} \beta$ for reported reverberation-mapped AGNs. In this paper, we find that there are some mapped AGNs, and virial $\mathrm{BH}$ masses based on $\mathrm{H} \alpha$ are much different from $\mathrm{BH}$ masses on $\mathrm{H} \beta$, which indicates some special information for the structure of emission line regions for Balmer lines. This paper is organized as follows. Section 2 shows the results based on the parameters (measured line width and size of the BLR based on longperiod variations of line emission and continuum emission) of reported reverberation-mapped AGNs. Section 3 gives the detailed results for the blue quasar PG $0052+251$ with very different virial $\mathrm{BH}$ masses on $\mathrm{H} \alpha$ and on $\mathrm{H} \beta$. Section 4 gives the discussion and conclusion. In this paper, the cosmological parameters $H_{0}=70 \mathrm{~km} \mathrm{~s}^{-1} \mathrm{Mpc}^{-1}, \Omega_{\Lambda}=0.7$, and $\Omega_{m}=0.3$ have been adopted.

\section{VIRIAL BH MASSES ON BALMER LINES FOR MAPPED AGNs}

As shown in the Introduction, we try to check virial $\mathrm{BH}$ masses on $\mathrm{H} \alpha$ and $\mathrm{H} \beta$ for mapped AGNs. The reported mapped AGNs are mainly included in two research groups, the AGNWATCH group (public data of 10 AGNs can be found at http://www.astronomy.ohio-state.edu/ agnwatch/) and the research group at the Wise Observatory at Tel Aviv University (public data of 17 PG quasars can be found at http://wise-obs.tau.ac.il/ shai/PG/; Kaspi et al. 2000). Corresponding references about observational techniques and instruments for the two groups can be found at the two Web sites above.

Before proceeding further, we roughly check results reported in the literature (especially Kaspi et al. 2000, 2005; Peterson et al. 2004; Bentz et al. 2010b) for all the 43 reverberationmapped AGNs whose observation data are either public or not. Here we mainly compare properties of broad $\mathrm{H} \alpha$ and broad $\mathrm{H} \beta$ (the two strongest broad emission lines in the optical band of AGNs), including line widths (second moment rather than FWHM, which is the best approximation of $V$ in
Equation (1) as supposed by Peterson et al. 2004) and the sizes of the BLR (the measured time lag between the line emission and continuum emission, not the calculated value from the empirical relation of $\left.R \propto L^{\sim 0.5}\right)$ based on $\mathrm{H} \alpha\left(R_{\mathrm{BLR}}^{\mathrm{H} \alpha}\right)$ and $\mathrm{H} \beta\left(R_{\mathrm{BLR}}^{\mathrm{H} \beta}\right)$, which are collected and listed in Table 1. Here, only objects with reliable parameters (line width and size of BLR), $P>1.5 \times P_{\text {err }}$ (where $P$ means the measured parameter and $P_{\text {err }}$ represents the corresponding uncertainty for the parameter) are collected from the literature. Based on this simple criterion, some reverberation-mapped objects should be rejected. For example, the size of NGC 3227's BLR based on $\mathrm{H} \beta$ is $R_{\mathrm{BLR}}^{\mathrm{H} \beta} \sim 8.2_{-8.4}^{+5.1}$ light days; the uncertainty of 8.4 is larger than the measured value 8.2, and thus NGC 3227 is rejected. We also should note that the famous reverberationmapped object NGC 5548 is rejected due to the following reasons. On the one hand, in Peterson et al. (2004), the line width of the broad $\mathrm{H} \alpha$ is not reliable due to larger uncertainty. On the other hand, we find that the sizes of the BLRs are similar, but the line widths of the broad $\mathrm{H} \beta$ (both second moment and FWHM) are much different in Peterson et al. (2004) from those shown in Bentz et al. (2010b), $\sigma_{\mathrm{H} \beta} \sim 2000 \mathrm{~km} \mathrm{~s}^{-1}$ and $\operatorname{FWHM}(\mathrm{H} \beta) \sim 5800 \mathrm{~km} \mathrm{~s}^{-1}$ in Peterson et al. (2004), $\sigma_{\mathrm{H} \beta} \sim 4200 \mathrm{~km} \mathrm{~s}^{-1}$ and $\operatorname{FWHM}(\mathrm{H} \beta) \sim 12,000 \mathrm{~km} \mathrm{~s}^{-1}$ in Bentz et al. (2010b). Thus, NGC 5548 is not included in our parent sample listed in Table 1. Furthermore, there is another object, PG 0844, we should note. For PG 0844, there are reliable parameters of the line width and size of the BLR for broad $\mathrm{H} \alpha$ and broad $\mathrm{H} \beta$ in Kaspi et al. (2000); however, there is no reliable size of the BLR based on $\mathrm{H} \beta$ in Peterson et al. (2004). Thus, PG 0844 is also rejected. Eventually, there are 16 objects listed in Table 1: 9 objects have public spectra (7 PG quasars observed by the Wise Observatory and 2 objects included in the AGNWATCH project) and the other 7 objects have no public spectra. Furthermore, we should note that the listed values of parameters in Table 1 are values from more recent literature. For example, values for PG 0052 can be found in Kaspi et al. (2000) and Peterson et al. (2004). Then the listed values for PG 0052 are collected from Peterson et al. (2004).

As shown in the Introduction, it is clear that there is one strong correlation for mapped AGNs based on virial BH masses:

$$
\begin{aligned}
\sigma_{\mathrm{H} \alpha}^{2} \times R_{\mathrm{BLR}}^{\mathrm{H} \alpha} & =\sigma_{\mathrm{H} \beta}^{2} \times R_{\mathrm{BLR}}^{\mathrm{H} \beta} \\
\left(\frac{\sigma_{\mathrm{H} \alpha}}{\sigma_{\mathrm{H} \beta}}\right)^{2} & =\frac{R_{\mathrm{BLR}}^{\mathrm{H} \beta}}{R_{\mathrm{BLR}}^{\mathrm{H} \alpha}} .
\end{aligned}
$$

In this section, we will check the correlation for the selected mapped AGNs. Figure 1 shows the correlation between the line width ratio of broad $\mathrm{H} \beta$ to broad $\mathrm{H} \alpha\left(\sigma_{\mathrm{H} \beta}^{2} / \sigma_{\mathrm{H} \alpha}^{2}\right)$ and the size ratio of $R_{\mathrm{BLR}}^{\mathrm{H} \alpha}$ to $R_{\mathrm{BLR}}^{\mathrm{H} \beta}$ for the 16 reverberation-mapped objects listed in Table 1. For all the 16 objects, the Spearman rank correlation coefficient for the correlation is about 0.4 with $P_{\text {null }} \sim 14 \%$ (the two-sided significance of deviation from zero), which indicates there is one rough positive correlation. In this figure, we also show the corresponding $99 \%$ confidence bands for the linear correlation $\sigma_{\mathrm{H} \beta}^{2} / \sigma_{\mathrm{H} \alpha}^{2}=R_{\mathrm{BLR}}^{\mathrm{H} \alpha} / R_{\mathrm{BLR}}^{\mathrm{H} \beta}$. Based on the results in Figure 1, there are two probable outliers, PG 0052 and NGC 4253, which deviate from the linear correlation supported by the virialization method. After the two outliers are rejected, the recalculated Spearman rank correlation coefficient is about 0.9 with $P_{\text {null }} \sim 4 \times 10^{-5}$.

Based on the results shown in Figure 1, the much different virial $\mathrm{BH}$ masses based on $\mathrm{H} \alpha$ and $\mathrm{H} \beta$ should indicate that 


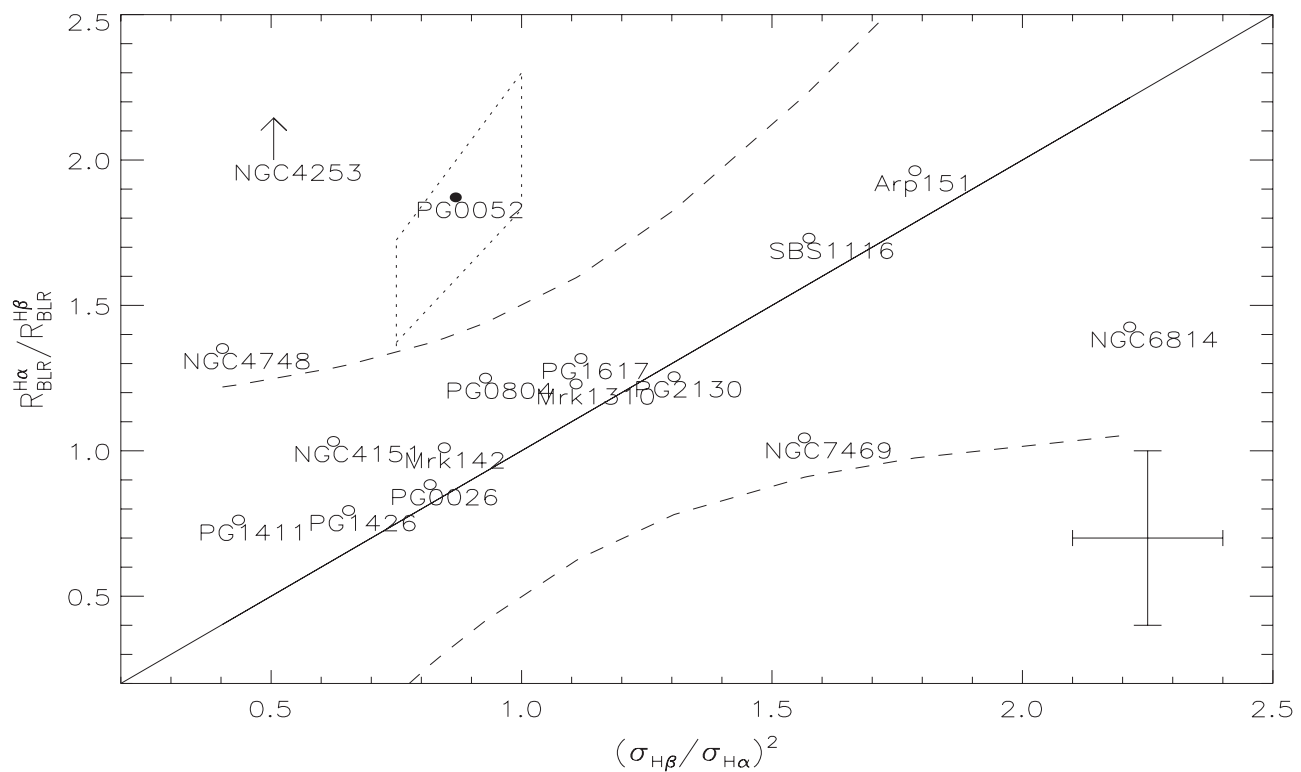

Figure 1. Correlation between the line width (second moment) ratio of $\sigma_{\mathrm{H} \beta}^{2} / \sigma_{\mathrm{H} \alpha}^{2}$ and the size ratio of $R_{\mathrm{BLR}}^{\mathrm{H} \alpha} / R_{\mathrm{BLR}}^{\mathrm{H} \beta}$. The standard error bar for data points is shown in the bottom right corner. Object PG $0052+251$ is marked with a solid circle. The area marked by dotted lines around PG $0052+251$ represents the estimated area in the plane, if the intermediate BLR was accepted for PG 0052 (results from Equation (6)). The solid line and the dashed line represent the correlation $\sigma_{\mathrm{H} \beta}^{2} / \sigma_{\mathrm{H} \alpha}^{2}=R_{\mathrm{BLR}}^{\mathrm{H} \alpha} / R_{\mathrm{BLR}}^{\mathrm{H} \beta}$ and its $99 \%$ confidence bands. For NGC 4253 , its true position $([0.51,4.1])$ is much farther away from the correlation. In order to clearly show the other objects, we use an upper arrow to represent that the true position of NGC 4253 should be much higher than the shown position [0.51,2].

Table 1

Parameters for the 16 Mapped AGNs

\begin{tabular}{|c|c|c|c|c|c|}
\hline Name & $\begin{array}{c}\sigma_{\mathrm{H} \alpha} \\
\left(\mathrm{km} \mathrm{s}^{-1}\right)\end{array}$ & $\begin{array}{c}R_{\mathrm{BLR}}^{\mathrm{H} \alpha} \\
\text { (Light Days) }\end{array}$ & $\begin{array}{c}\sigma_{\mathrm{H} \beta} \\
\left(\mathrm{km} \mathrm{s}^{-1}\right)\end{array}$ & $\begin{array}{c}R_{\mathrm{BLR}}^{\mathrm{H} \beta} \\
\text { (light-days) }\end{array}$ & Public \\
\hline PG 0026+129 & $1961 \pm 135$ & $98.1_{-25.5}^{+28.3}$ & $1773 \pm 285$ & $111.0_{-28.3}^{+24.1}$ & Kaspi \\
\hline PG $0052+251$ & $1913 \pm 85$ & $163.7_{-38.3}^{+58.5}$ & $1783 \pm 86$ & $89.8_{-24.1}^{+24.5}$ & Kaspi \\
\hline PG 0804+761 & $2046 \pm 138$ & $183.6_{-13.3}^{+15.3}$ & $1971 \pm 105$ & $146.9_{-18.9}^{+18.8}$ & Kaspi \\
\hline NGC 4151 & $2422 \pm 79$ & $3.2_{-1.7}^{+1.9}$ & $1914 \pm 42$ & $3.1 \pm 1.3$ & AGNWATCH \\
\hline PG $1411+442$ & $2437 \pm 196$ & $94.7_{-31.5}^{+36.0}$ & $1607 \pm 169$ & $124.3_{-61.7}^{+61.0}$ & Kaspi \\
\hline PG $1426+015$ & $4254 \pm 290$ & $75.5_{-32.5}^{+30.5}$ & $3442 \pm 308$ & $95.0_{-37.1}^{+29.9}$ & Kaspi \\
\hline PG $1617+175$ & $2483 \pm 160$ & $94.2_{-25.2}^{+19.1}$ & $2626 \pm 211$ & $71.5_{-33.7}^{+29.6}$ & Kaspi \\
\hline PG 2130+099 & $1421 \pm 80$ & $198.4_{-23.4}^{+32.6}$ & $1623 \pm 86$ & $158.1_{-18.7}^{+29.8}$ & Kaspi \\
\hline NGC 7469 & $1164 \pm 68$ & $4.7_{-1.3}^{+1.6}$ & $1456 \pm 207$ & $4.5_{-0.8}^{+0.7}$ & AGNWATCH \\
\hline Mrk142 & $934 \pm 61$ & $2.90_{-0.92}^{+1.22}$ & $859 \pm 102$ & $2.87_{-0.87}^{+0.76}$ & NO \\
\hline SBS1116+583A & $1218_{-99}^{+147}$ & $4.12_{-0.98}^{+1.41}$ & $1528 \pm 184$ & $2.38_{-0.51}^{+0.64}$ & NO \\
\hline Arp151 & $937 \pm 34$ & $8.01_{-1.00}^{+1.05}$ & $1252 \pm 46$ & $4.08_{-0.69}^{+0.50}$ & NO \\
\hline Mrk1310 & $717 \pm 75$ & $4.60_{-0.62}^{+0.67}$ & $755 \pm 138$ & $3.74_{-0.62}^{+0.60}$ & NO \\
\hline NGC 4253 & $726 \pm 35$ & $25.50_{-0.86}^{+0.66}$ & $516 \pm 218$ & $6.24_{-1.24}^{+1.65}$ & NO \\
\hline NGC 4748 & $1035 \pm 74$ & $7.61_{-4.64}^{+3.01}$ & $657 \pm 91$ & $5.63_{-2.25}^{+1.64}$ & NO \\
\hline NGC 6814 & $1082 \pm 52$ & $9.51_{-1.56}^{+1.91}$ & $1610 \pm 108$ & $6.67_{-0.90}^{+0.88}$ & NO \\
\hline
\end{tabular}

Notes. "Kaspi" means that the public observed spectra can be found from Web site http://wise-obs.tau.ac.il/ shai/PG/. "AGNWATCH" means the public observed spectra can be found from the AGNWATCH project, http://www.astronomy.ohio-state.edu/ agnwatch/. "NO" means there are no public observed spectra; the listed parameters are collected from Bentz et al. (2010b). The sizes of the BLR based on broad Balmer emission lines are the ones determined by $\tau_{\text {cent }}$, and line widths are the ones measured from the rms spectra as discussed in Peterson et al. (2004). The listed values of parameters are values from more recent literature.

there are some unique characteristics for broad Balmer emission line regions. To find and study the characteristics are the main objectives of the following section. For the two outliers, only PG 0052 has public observed spectra, which can be downloaded from http://wise-obs.tau.ac.il/ $\sim$ shai/PG/. In the following section, we will mainly study the properties of the reverberation-mapped AGN PG 0052 and try to find some special characteristics of the broad Balmer line regions of PG 0052.

\section{MAIN RESULTS FOR PG 0052}

In this paper, all the observational data and spectra of PG $0052+251$ are collected from Kaspi et al. (2000; 


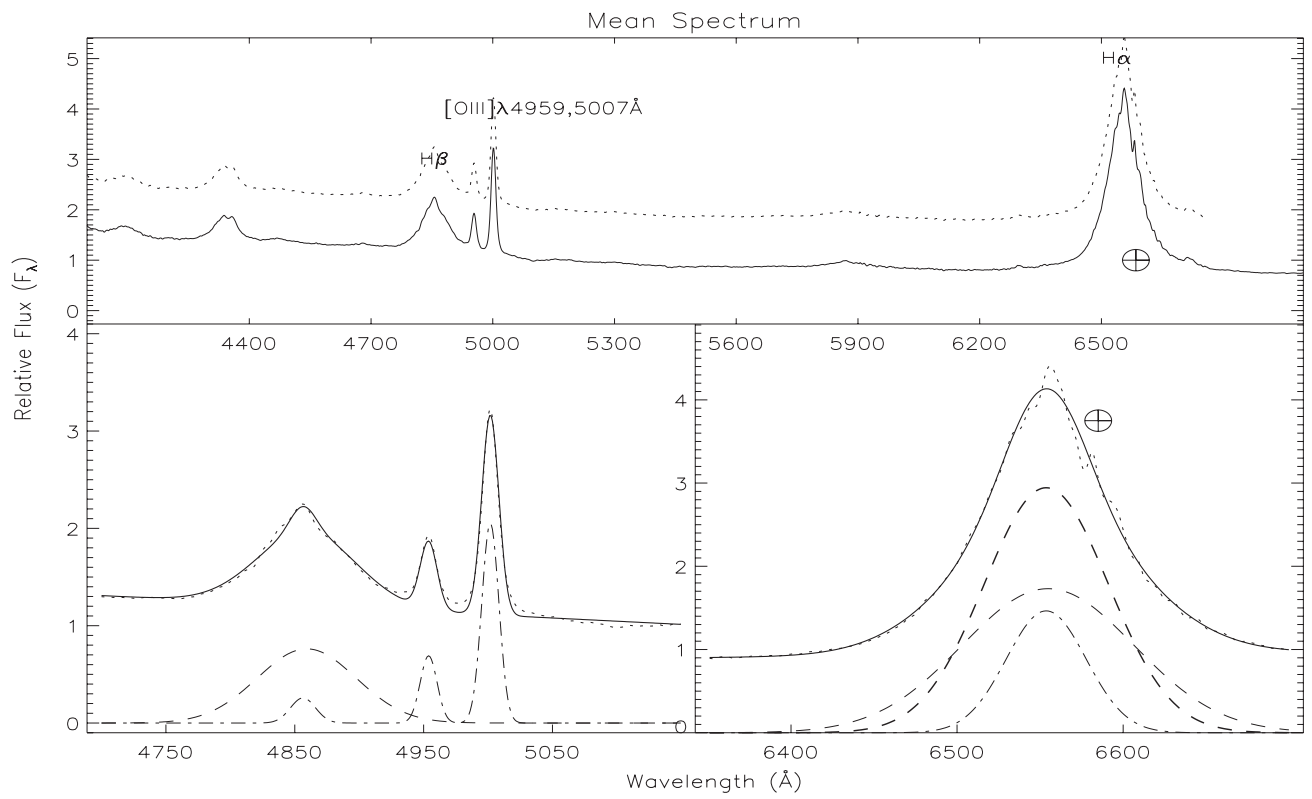

Figure 2. Mean spectrum of PG $0052+251$ and best-fit results for emission lines around $\mathrm{H} \alpha$ and $\mathrm{H} \beta$. In the top panel, the solid line shows our mean spectrum created using the PCA method (flux $(5100 \AA)=1)$ and the dotted line shows the mean spectrum shown in Kaspi et al. $(2000 ;$; flux $(5100 \AA)=2)$. In the bottom left panel, the dotted line shows the mean spectrum around $\mathrm{H} \beta$, the thick solid line represents the best-fit results, the dashed line represents the broad component of $\mathrm{H} \beta$ fitted by one broad Gaussian function, and the dot-dashed line shows narrow components of $\mathrm{H} \beta$ and [O III] $\lambda 4959,5007 \AA$. In the bottom right panel, the dotted line shows the mean spectrum around $\mathrm{H} \alpha$ and the thick solid line represents the best-fit results. The thick dashed line shows the broad component, if one broad Gaussian function is applied to fit $\mathrm{H} \alpha$. The thin dashed line shows the inner broad component and the dot-dashed line shows the intermediate broad component, if the two broad Gaussian functions are applied to fit $\mathrm{H} \alpha$. The symbols " $\Theta$ " in the figure show the positions of the features of the atmospheric $A$ band near $7620 \AA$ in the observed frame.

http://wise-obs.tau.ac.il/ shai/PG/). A detailed description of the data and spectra can be found in Kaspi et al. (2000). Here, we do not describe instruments and observational techniques. There are 56 spectra from $4000 \AA$ to $8000 \AA$ with a dispersion of $\sim 3.8 \AA$ pixel $^{-1}$ and a spectral resolution of about $\sim 10 \AA$ observed from 1991 October 16 to 1998 September 27 for PG $0052+251$. However, there is one spectrum with many bad pixels around $\mathrm{H} \alpha$; thus, only 55 spectra are considered. The collected spectra from the above Web site have been binned into $1 \AA$ per pixel and have been padded from $3000 \AA$ to $9000 \AA$, as shown at the Web site. We mainly consider the data and spectra as follows.

\subsection{Properties of the Mean Spectrum}

The mean spectrum of PG $0052+251$ can be created using the principal component analysis (PCA) technique or the Karhunen-Loeve transform method, applied for observed noisy spectra. The PCA technique is a mathematical procedure that uses an orthogonal transformation to convert a set of observations of possibly correlated variables into a set of values of uncorrelated variables called principal components. Certainly, mean subtraction (or mean centering) is necessary for performing PCA to ensure that the first principal component describes the direction of maximum variance. However, if mean subtraction is not performed, the first eigencomponent through the PCA technique commonly represents the mean spectrum of noisy spectra. Here, the convenient and public IDL PCA program "pca_solve.pro" written by D. Schlegel at Princeton University is used, which is included in the Sloan Digital Sky Survey software package of IDLSPEC2D (http://spectro.princeton.edu/).

Figure 2 shows the mean spectrum of PG 0052+251 with relative flux density flux $(5100 \AA)=1$. Furthermore, the mean spectrum of PG $0052+251$ created by Kaspi et al. (2000) is also shown in Figure 2, which is the same as our mean spectrum created using the PCA method. Then, Balmer emission lines are fitted by simple Gaussian functions through the Levenberg-Marquardt least-squares minimization method. The best-fit results are also shown in Figure 2. Emission lines around $\mathrm{H} \beta$ can be best fitted by one broad Gaussian function for broad $\mathrm{H} \beta$ and three narrow Gaussian functions for narrow $\mathrm{H} \beta$ and $[\mathrm{O}$ III] $\lambda 4959,5007 \AA$ doublet. Here when the [O III] doublet is fitted, we require that $[\mathrm{O}$ III] $\lambda 4959,5007 \AA$ have the same line width in units of $\mathrm{km} \mathrm{s}^{-1}$, and the flux ratio of $\left[\mathrm{O}_{\text {III }}\right] \lambda 4959 \AA$ to $\left[\mathrm{O}_{\text {III }}\right] \lambda 5007 \AA$ is the theoretical value 0.33 (Dimitrijevic et al. 2007). Emission lines around $\mathrm{H} \alpha$ can be fitted by one broad Gaussian function. Here we do not consider narrow emission lines around $\mathrm{H} \alpha$ in the mean spectrum due to much weaker [O I] $\lambda 6300,6363,\left[\mathrm{~N}_{\mathrm{II}}\right] \lambda 6548,6583$, and [S II] $\lambda 6716$, 6731 doublets. Furthermore, we should note that features of the atmospheric $A$ band near $7620 \AA$ in the observed frame can be detected, which are marked in Figure 2 and in the following Figure 4 by the symbol " $\oplus$ ". Here, we simply discuss effects of the features on measured line parameters. Line parameters around $\mathrm{H} \alpha$ are first measured without any consideration of the effects of the features (functions are applied to total observed line profile), and then remeasured with consideration of the effects of the features (functions are applied to the observed line profile with features of the atmospheric $A$ band rejected). The measured line parameters due to the two different procedures are similar. Thus, in the following part, the effects of the features of the atmospheric $A$ band near $7620 \AA$ are totally ignored. The measured line parameters of broad $\mathrm{H} \alpha$ and broad $\mathrm{H} \beta$ in the mean spectrum are listed in Table 2.

Based on the line parameters listed in Table 2, it is clear that the line width (second moment) of the broad $\mathrm{H} \beta\left(\sigma_{\mathrm{H} \beta} \sim\right.$ $38 \AA \sim 2370 \mathrm{~km} \mathrm{~s}^{-1}$ ) is much different from the line width of the broad $\mathrm{H} \alpha\left(\sigma_{\mathrm{H} \alpha} \sim 37 \AA \sim 1700 \mathrm{~km} \mathrm{~s}^{-1}\right)$, which is much different from the results shown in Greene \& Ho (2005) and Korista \& Goad (2004; $\left.\sigma_{\mathrm{H} \beta} / \sigma_{\mathrm{H} \alpha} \sim 1.06-1.1\right)$. The results indicate that 


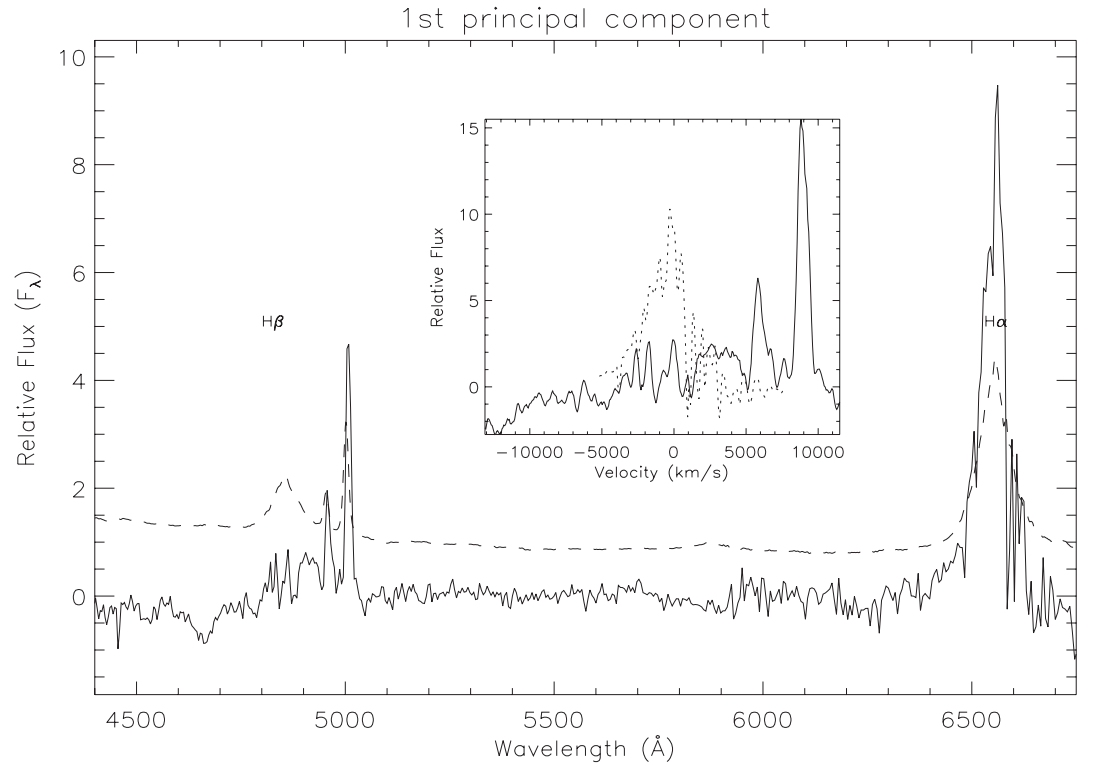

Figure 3. Properties of line cores. The solid line represents the first PCA component based on the observed spectra with zero mean. The dashed line represents the mean spectrum based on the observed spectra shown in Figure 2. The middle panel shows the comparison of the line cores of $\mathrm{H} \alpha$ and $\mathrm{H} \beta$. In the panel, the solid line represents the line core of $\mathrm{H} \beta$ with a flux density scaled by 3 and the dotted line represents the line core of $\mathrm{H} \alpha$.

Table 2

Parameters of PG $0052+251$

\begin{tabular}{lccccc}
\hline \hline Line & $\begin{array}{c}\sigma \\
(\AA)\end{array}$ & $\begin{array}{c}\text { Flux } \\
10^{-16}\left(\mathrm{erg} \mathrm{s}^{-1} \mathrm{~cm}^{-2}\right)\end{array}$ & $\begin{array}{c}R_{\mathrm{BLR}}(p) \\
\text { (light-days) }\end{array}$ & $\begin{array}{c}R_{\mathrm{BLR}}(c) \\
\text { (light-days) }\end{array}$ & $R_{\mathrm{CCF}}(\max )$ \\
\hline $\mathrm{H} \beta$ (broad) & 38.4 & 1426.3 & $109 \pm 25$ & $111 \pm 15$ & 0.76 \\
$\mathrm{H} \alpha($ tot broad $)$ & 37.4 & 9612.5 & $191 \pm 24$ & $214 \pm 19$ & 0.72 \\
$\mathrm{H} \alpha($ inner broad $)$ & 51.8 & 5067.9 & $110 \pm 20$ & $116 \pm 16$ & 0.68 \\
$\mathrm{H} \alpha($ int broad $)$ & 24.1 & $1649.5^{\star}$ & $703 \pm 45$ & $678 \pm 40$ & 0.38 \\
\hline
\end{tabular}

Notes. $\mathrm{H} \alpha$ (tot broad) means the observed total broad $\mathrm{H} \alpha$. $\mathrm{H} \alpha$ (inner broad) means the inner broad component of $\mathrm{H} \alpha$. $\mathrm{H} \alpha$ (int broad) represents the intermediate broad component of $\mathrm{H} \alpha$. The second column gives the second moment of the broad component in units of $\AA$ shown in the mean spectrum of PG $0052+251$, the third column shows the measured mean flux density of the broad component for 55 observed spectra of PG $0052+251$, the fourth column shows the measured size of the BLR through the peak value of the CCF results, the fifth column shows the measured size of the BLR through the central value of the CCF results, and the sixth column shows the maximum coefficient through the CCF. The corresponding error of the measured size of the BLR is determined through the bootstrap method as shown in Figure 5. $1649.5^{\star}$ means the mean value is not that reliable and is probably lower than the internal value due to a very weak intermediate broad $\mathrm{H} \alpha$ in some observed spectra.

broad Balmer line regions of PG 0052 probably have some special characteristics that are different from common quasars. In other words, a single, broad Gaussian function applied to fit broad $\mathrm{H} \alpha$ and $\mathrm{H} \beta$ is not that good. Thus, we consider broad Balmer emission lines using a different method as follows.

\subsection{Properties of Line Cores}

In order to more clearly show the properties of broad Balmer emission lines, the PCA technique is applied again as has been done in Brotherton et al. (1994) and Francis et al. (1992). Before studying properties of line cores, the commonly accepted step of mean subtraction (or mean centering) is first performed. Then, after the PCA technique is applied to the spectra with zero mean, the first principal component represents emission line cores (Brotherton et al. 1994; Francis et al. 1992). Figure 3 shows the emission line cores around $\mathrm{H} \beta$ and $\mathrm{H} \alpha$. In the figure, the line profile of $\mathrm{H} \beta$ with a flux density scaled by 3 (the intrinsic flux ratio of $\mathrm{H} \alpha$ to $\mathrm{H} \beta$ for a blue quasar) is directly compared with the line profile of $\mathrm{H} \alpha$ in the first principal component. Based on the results shown in Figure 3, it is clear that the line cores of $\mathrm{H} \beta$ and $\mathrm{H} \alpha$ are very different; there is one apparent broad component in $\mathrm{H} \alpha$ but no broad component in $\mathrm{H} \beta$ in the first principal component. The results indicate that aside from the similar inner broad component similar to that of observed broad $\mathrm{H} \beta$, there is one other intermediate broad component in $\mathrm{H} \alpha$ but no intermediate broad component in $\mathrm{H} \beta$ (one seriously obscured intermediate component for Balmer lines). Moreover, we should note that there are narrow lines around $\mathrm{H} \beta$ in the first principal component shown in Figure 3, which is due to a much extended component of $[\mathrm{O}$ III $] \lambda 4959,5007$. Similar narrow lines can also be found in the rms spectrum of PG 0844 shown in Figure 2 in Kaspi et al. (2000). Those narrow lines cannot affect our results about the line cores of $\mathrm{H} \alpha$ and $\mathrm{H} \beta$.

Thus, broad $\mathrm{H} \alpha$ is fitted again by two Gaussian functions, one broad Gaussian function with a similar line width to that of the broad $\mathrm{H} \beta$ and one intermediate broad Gaussian function, through the Levenberg-Marquardt least-squares minimization method. The two components are also shown in the bottom right panel of Figure 2. The parameters of the two components of $\mathrm{H} \alpha$ are also listed in Table 2. The line width (second moment) of the intermediate broad component of $\mathrm{H} \alpha$ is about $1100 \mathrm{~km} \mathrm{~s}^{-1}$ which is much larger than the line width (second moment) of 
narrow $[\mathrm{O} \mathrm{III}] \lambda 5007\left(\sigma([\mathrm{O} \mathrm{III}]) \sim 6.6 \AA=395 \mathrm{~km} \mathrm{~s}^{-1}\right)$. Based on the flux ratio of the inner broad $\mathrm{H} \alpha$ to intermediate broad $\mathrm{H} \alpha, \sim 2.6-3$, it is clear that the intermediate broad component of $\mathrm{H} \alpha$ is distinct and clearly decomposed in the mean spectrum of PG $0052+251$. Certainly, we also try to fit the broad $\mathrm{H} \beta$ by two broad Gaussian functions. However, the intermediate broad component of $\mathrm{H} \beta$ is not reliable due to a smaller measured line width and line luminosity than the corresponding measured uncertainties for the expected intermediate component of $\mathrm{H} \beta$, which is consistent with the results shown in Figure 3. The results indicate that the intermediate broad component of BLR probably exists and is seriously obscured (no intermediate broad component of $\mathrm{H} \beta$ ) for PG $0052+251$. Certainly, the intermediate broad component of $\mathrm{H} \beta$ cannot be detected, perhaps due to the low quality of spectra for PG $0052+251$. Although there is no true value of the flux ratio of the intermediate broad component of $\mathrm{H} \alpha$ to the intermediate broad component of $\mathrm{H} \beta$, it is not difficult to determine the cut-off value of signal to noise $(\mathrm{S} / \mathrm{N})$ for future high quality spectra. If we accept that the flux ratio of the intermediate broad component of $\mathrm{H} \alpha$ to the intermediate broad component of $\mathrm{H} \beta$ is about 6 , the $\mathrm{S} / \mathrm{N}$ should be larger than 24.

Before the end of this subsection, we discuss properties of $\chi^{2}$ for the fitted results of $\mathrm{H} \alpha$ and $\mathrm{H} \beta$, in order to find more reliable evidence for the existence of the second Gaussian component for H $\alpha$. Here $\chi^{2}$ is the value of the summed, squared residuals for the measured parameters divided by the degrees of freedom, which can be used as one good indicator to determine whether the fitted results are acceptable. Here, we accept that the uncertainty for the flux is about $\sim 10 \%$. The values of $\chi^{2}$ are $0.18,0.06$, and 0.08 for best-fit results by one Gaussian function, by two Gaussian functions for $\mathrm{H} \alpha$, and by Gaussian functions for lines around $\mathrm{H} \beta$, respectively. Then, based on fitted results by one or two Gaussian functions for $\mathrm{H} \alpha$, the $F$ test is performed: at the $99 \%$ confidence level, the calculated variance ratio of results by one Gaussian function to results by two Gaussian functions is 2.14 for $\mathrm{H} \alpha$ in the mean spectrum, which is much larger than the $F$ value 1.34 . The results indicate that two Gaussian functions for $\mathrm{H} \alpha$ are necessary. Due to the unreliable second Gaussian component for $\mathrm{H} \beta$ through the leastsquares minimization method, the $F$-test is not performed for results of $\mathrm{H} \beta$.

\subsection{Properties of the Observed Spectra}

We consider the observed spectra collected from Kaspi et al. (2000) in this subsection. Line parameters are measured through Gaussian functions for all the 55 observed spectra with both $\mathrm{H} \alpha$ and $\mathrm{H} \beta$ for $\mathrm{PG} 0052+251$, as done above for the Balmer lines in the mean spectrum. Emission lines around $\mathrm{H} \beta$ are measured once: only a single broad component is fitted $\mathrm{H} \beta$. The emission line $\mathrm{H} \alpha$ is measured twice, (1) one broad Gaussian function is fitted to $\mathrm{H} \alpha$, (2) two broad Gaussian functions are fitted to $\mathrm{H} \alpha$ : one broad component and the other intermediate broad component as shown in the subsection above. When two Gaussian functions are applied to fit $\mathrm{H} \alpha$, the broad component has a similar line width to the one of broad $\mathrm{H} \beta$ (with a permitted scatter of $0.1 \mathrm{dex}$ ) and the line width ratio of the corresponding two functions (broad to intermediate broad) is fixed to $2.16\left(\sim\left(2370 \mathrm{~km} \mathrm{~s}^{-1} / 1100 \mathrm{~km} \mathrm{~s}^{-1}\right)\right.$, based on the measured line widths of two components of $\mathrm{H} \alpha$ in the mean spectrum).

After the measurements of the line parameters, we find that if one Gaussian function is applied to fit $\mathrm{H} \alpha$, the line width ratio of the total broad $\mathrm{H} \beta$ to the total broad $\mathrm{H} \alpha$ is about $1.4 \pm 0.1$, which is much larger than the mean value of $\sim 1.1$ for quasars shown in Greene \& Ho (2005) and larger than the theoretical value of $\sim 1.06-1.1$ in Korista \& Goad (2004). Furthermore, if one Gaussian function is applied to fit $\mathrm{H} \alpha$, the flux ratio of the total broad $\mathrm{H} \alpha$ to the total broad $\mathrm{H} \beta$ (Balmer decrement) is about 6.8, which is a larger and more unreasonable value for the blue quasar PG 0052+251 (the mean value from composite spectra of quasar is about 3.56 in Vanden Berk et al. 2001). However, if two Gaussian functions are applied to fit $\mathrm{H} \alpha$, the flux ratio of the inner broad $\mathrm{H} \alpha$ to the inner broad $\mathrm{H} \beta$ (the observed total broad $\mathrm{H} \beta$ only includes the inner broad $\mathrm{H} \beta$; the intermediate broad $\mathrm{H} \beta$ is seriously obscured) is about 3.61 , which is consistent with the value from the composite spectra of quasars (Vanden Berk et al. 2001), and the line width ratio is about $\sigma_{\mathrm{H} \alpha} / \sigma_{\mathrm{H} \beta} \sim 0.94$ (here $\mathrm{H} \alpha$ is the one without the intermediate broad $\mathrm{H} \alpha$ ), which is consistent with the result shown in Greene \& Ho (2005) and Korista \& Goad (2004); H $\beta$ is slightly larger than $\mathrm{H} \alpha\left(\sigma_{\mathrm{H} \alpha} / \sigma_{\mathrm{H} \beta} \sim 0.91\right)$. Certainly, there are some cases where intermediate broad $\mathrm{H} \alpha$ is too weak to be detected in the observed spectra, as shown in Figure 4, which is probably due to the observed spectra with lower resolution. It is clear that the total observed broad $\mathrm{H} \alpha$ separated into two components (one inner broad component and one intermediate broad component) should be more reasonable for the blue quasar PG 0052+251.

\subsection{CCF Results}

Finally, we consider the results from the cross-correlation function (CCF) applied to measure the size of the BLR of PG $0052+251$, which can be determined by the time lag between the variations of continuum emission and the variations of broadline emission. Here the variations of broad emission lines have four components: variations of the observed broad $\mathrm{H} \beta$ (inner broad $\mathrm{H} \beta$ ), variations of the observed total broad $\mathrm{H} \alpha$, variations of the inner broad $\mathrm{H} \alpha$ (coming from inner BLR), and variations of the intermediate broad $\mathrm{H} \alpha$ (coming from intermediate BLR). Here the flux densities of the total broad $\mathrm{H} \alpha, \mathrm{H} \beta$, and continuum emission used are collected from Kaspi et al. (2000). And then, according to the measured flux ratio of the broad $\mathrm{H} \alpha$ to the intermediate broad $\mathrm{H} \alpha$ of each observed spectrum, the corrected flux density of the total broad $\mathrm{H} \alpha$ for each observed spectrum can be separated into two values for the inner broad component and for the intermediate broad component of $\mathrm{H} \alpha$. Thus, the effects from different instruments in different configurations can be totally ignored (van Groningen \& Wanders 1992). In other words, the following used flux densities of broad line components are reliable.

Here, the common interpolated cross-correlation function (ICCF; Gaskell \& Sparke 1986; Gaskell \& Peterson 1987; Peterson 1993) is applied to quantify the time lag between the continuum emission and the broad lines emission. We do not consider the $z$-transfer discrete correlation function (ZDCF; Alexander 1997; Edelson \& Krolik 1988; White \& Peterson 1994) any more, because the results from ZDCF are in excellent agreement with the results from ICCF (Peterson et al. 1991, 1992, 2004; Kaspi et al. 2000; Bentz et al. 2010b). Figure 5 shows the final results based on the four broad components of $\mathrm{H} \alpha$ and $\mathrm{H} \beta$, including the measured sizes of the BLR and the corresponding uncertainties determined by the bootstrap method (Press et al. 1992; Peterson et al. 1998). Measured sizes of the BLR based on variations of different broad components are listed in Table 2. The size of the inner BLR is about 110 light 


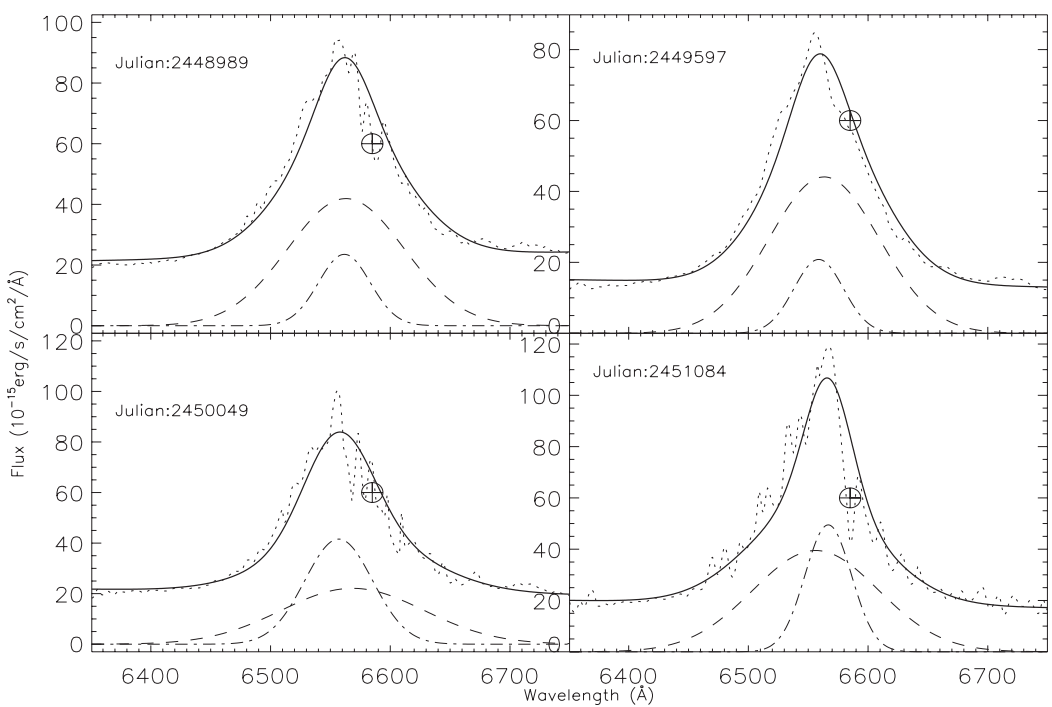

Figure 4. Best-fit results for observed $\mathrm{H} \alpha$. In each panel, the dotted line shows the observed spectrum, the thick solid line represents the best-fit results, the dashed line represents the inner broad component of $\mathrm{H} \alpha$, and the dot-dashed line shows the intermediate broad component of $\mathrm{H} \alpha$. In the case of Julian:2449596 (top right panel), there is no apparent intermediate broad component. The symbols " $\oplus$ " in the figure show the positions of the features of the atmospheric $A$ band near $7620 \AA$ in the observed frame.

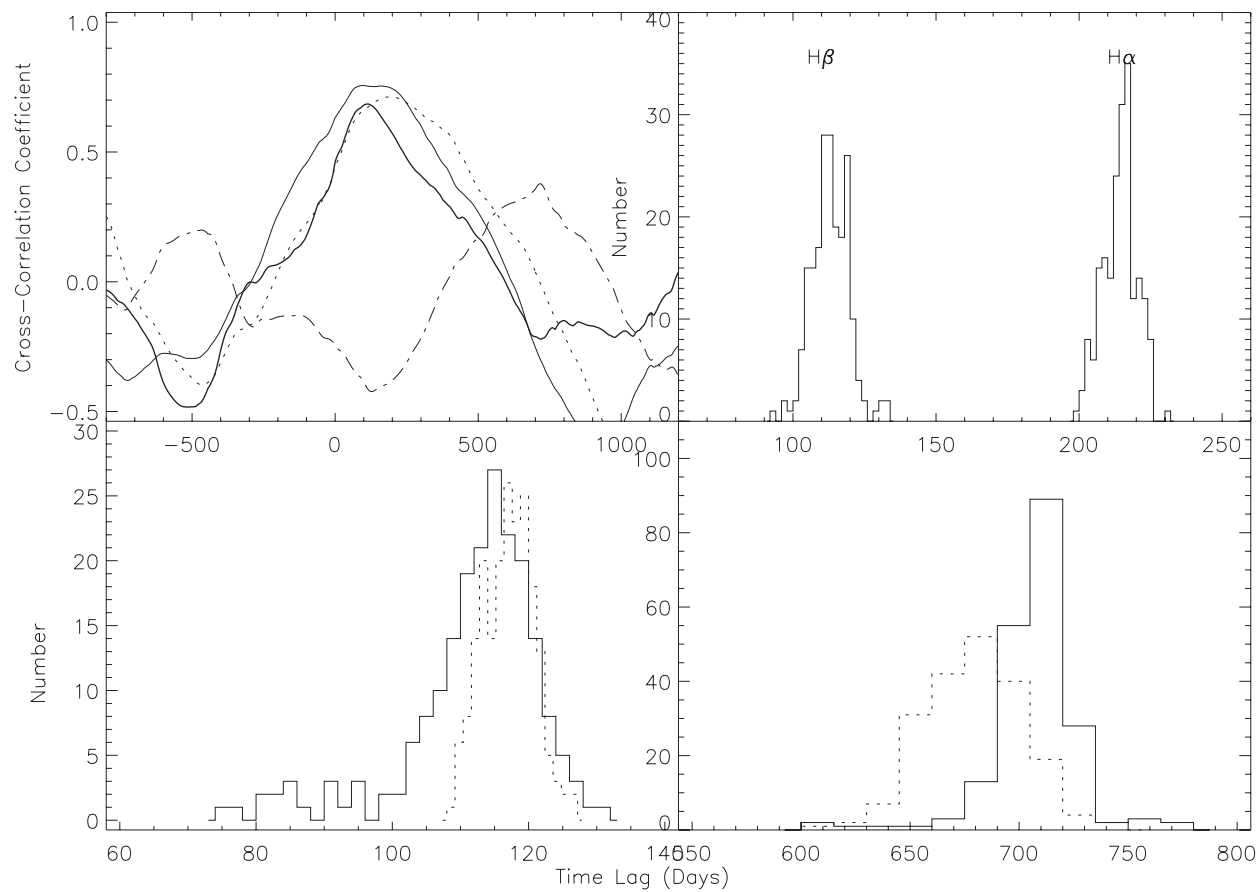

Figure 5. Top left panel shows results for CCF. The thin solid line represents the result for broad $\mathrm{H} \beta\left(\mathrm{CCF}\left(\mathrm{H} \beta, \operatorname{con}_{5100} \AA\right)\right)$. The dotted line represents the result for the total broad $\mathrm{H} \alpha\left(\mathrm{CCF}\left(\mathrm{H} \alpha_{\text {tot }}, c n_{5100} \AA\right)\right)$. The thick solid line represents the result for the inner broad $\mathrm{H} \alpha\left(\mathrm{CCF}\left(\mathrm{H} \alpha_{\text {inner broad }}, \operatorname{con}_{5100} \AA\right)\right)$. The dot-dashed line shows the result for intermediate broad $\mathrm{H} \alpha\left(\mathrm{CCF}\left(\mathrm{H} \alpha\right.\right.$ int broad, $\left.\left.\operatorname{con}_{5100} \AA\right)\right)$. The top right panel shows the distributions of time lags between the total broad Balmer emission and AGN continuum emission through the bootstrap method, in order to compare our results with that of Kaspi et al. (2000) and Peterson et al. (2004). The bottom left panel shows the distributions of time lag between the inner broad $\mathrm{H} \alpha$ emission (after the subtraction of the intermediate broad $\mathrm{H} \alpha$ ) and AGN continuum emission through the bootstrap method. The bottom right panel shows the distributions of time lag between intermediate broad H $\alpha$ emission and AGN continuum emission through the bootstrap method. In the two bottom panels, the solid line represents the distributions for time lag determined by peak values of CCF results and the dotted line represents the distributions of time lag determined by the central values of the CCF results.

days based on the inner broad $\mathrm{H} \alpha$ (or observed broad $\mathrm{H} \beta$ ) and the size of the intermediate BLR based on the intermediate broad $\mathrm{H} \alpha$ is about 700 light days. Furthermore, the measured sizes based on the observed total broad $\mathrm{H} \alpha$ and $\mathrm{H} \beta$ are consistent with the reported values in Kaspi et al. (2000, 2005) and Peterson et al. (2004).

The clear size of the intermediate broad component of $\mathrm{H} \alpha$ through the CCF results indicates that the intermediate BLR for $\mathrm{H} \alpha$ PG 0052 can be mathematically determined. Based on this result, we try to discuss some unique characteristics based on the intermediate BLR.

\subsection{Intermediate BLR}

Before giving some clear and further conclusions for mapped AGN PG 0052, we discuss the effects of the intermediate BLR on virial $\mathrm{BH}$ masses as follows.

There are two parameters used for virial $\mathrm{BH}$ masses in Equation (1), line width and the size of the BLR. The two 
parameters are based on observational information. If the intermediate broad component was accepted for $\mathrm{H} \alpha$ of PG 0052 , then the virial $\mathrm{BH}$ mass estimated from the properties of the total observed broad $\mathrm{H} \alpha$ (one inner broad component plus one intermediate broad component) should be different from the $\mathrm{BH}$ mass estimated from the inner (or intermediate) broad component, and that the estimated virial $\mathrm{BH}$ mass based on only the inner (or intermediate) broad component of $\mathrm{H} \alpha$ should be more accurate than the virial $\mathrm{BH}$ mass based on the total observed broad $\mathrm{H} \alpha$. Under the assumption of the intermediate BLR accepted for $\mathrm{H} \alpha$ of PG 0052, we would find that

$$
\begin{aligned}
\sigma_{\mathrm{H} \alpha}^{2} & \simeq f_{1} \times \sigma_{\mathrm{H} \alpha_{1}}^{2}+f_{2} \times \sigma_{\mathrm{H} \alpha_{2}}^{2} \\
R_{\mathrm{BLR}}^{\mathrm{H} \alpha} & \simeq f_{1} \times R_{\mathrm{BLR}}^{\mathrm{H} \alpha_{1}}+f_{2} \times R_{\mathrm{BLR}}^{\mathrm{H} \alpha_{2}},
\end{aligned}
$$

where $\sigma_{\mathrm{H} \alpha_{1}}\left(R_{\mathrm{BLR}}^{\mathrm{H} \alpha_{1}}\right)$ and $\sigma_{\mathrm{H} \alpha_{2}}\left(R_{\mathrm{BLR}}^{\mathrm{H} \alpha_{2}}\right)$ are the mean measured line widths (sizes of BLR) based on the inner and intermediate broad components of $\mathrm{H} \alpha$, and $f_{1}$ and $f_{2}$ represent parameters of flux weight: the flux ratio of the inner (intermediate) broad component to total observed broad $\mathrm{H} \alpha(f 1+f 2=1)$. One simple but clear method for proving the above equation can be found in the Appendix.

It is very interesting that for PG 0052 (only the inner broad component for $\mathrm{H} \beta$ but the inner broad plus intermediate broad component for $\mathrm{H} \alpha$ ), the virial $\mathrm{BH}$ masses based on the total observed broad $\mathrm{H} \alpha$ should depend on properties of the inner and the intermediate component of $\mathrm{H} \alpha$ :

$$
\begin{aligned}
M_{\mathrm{BH}} & \propto \sigma_{\mathrm{H} \alpha}^{2} \times R_{\mathrm{BLR}}^{\mathrm{H} \alpha} \\
& \propto\left(f_{1}^{2}+f_{2}^{2}\right)\left(\sigma_{\mathrm{H} \alpha_{1}}^{2} \times R_{\mathrm{BLR}}^{\mathrm{H} \alpha_{1}}\right) \\
& +f_{1} f_{2}\left(\sigma_{\mathrm{H} \alpha_{1}}^{2} \times R_{\mathrm{BLR}}^{\mathrm{H} \alpha_{2}}+\sigma_{\mathrm{H} \alpha_{2}}^{2} \times R_{\mathrm{BLR}}^{\mathrm{H} \alpha_{1}}\right) .
\end{aligned}
$$

In the equation above, the relation $\sigma_{\mathrm{H} \alpha_{1}}^{2} \times R_{\mathrm{BLR}}^{\mathrm{H} \alpha_{1}} \simeq \sigma_{\mathrm{H} \alpha_{2}}^{2} \times R_{\mathrm{BLR}}^{\mathrm{H} \alpha_{2}}$ is accepted (the $\mathrm{BH}$ mass estimated through properties of the inner broad $\mathrm{H} \alpha$ should be similar to the mass through properties of the intermediate broad $\mathrm{H} \alpha$ ). Then, we can compare $\mathrm{BH}$ masses based on the observed total broad $\mathrm{H} \beta$ (the inner broad component for $\mathrm{H} \beta$ ) and virial $\mathrm{BH}$ masses based on the observed total broad $\mathrm{H} \alpha$ :

$$
\frac{\sigma_{\mathrm{H} \alpha}^{2} \times R_{\mathrm{BLR}}^{\mathrm{H} \alpha}}{\sigma_{\mathrm{H} \beta}^{2} \times R_{\mathrm{BLR}}^{\mathrm{H} \beta}} \simeq\left(f_{1}^{2}+f_{2}^{2}\right)+f_{1} f_{2}\left(\frac{R_{\mathrm{BLR}}^{\mathrm{H} \alpha_{1}}}{R_{\mathrm{BLR}}^{\mathrm{H} \alpha_{2}}}+\frac{R_{\mathrm{BLR}}^{\mathrm{H} \alpha_{2}}}{R_{\mathrm{BLR}}^{\mathrm{H} \alpha_{1}}}\right) .
$$

In the equation above, the relation $\sigma_{\mathrm{H} \beta}^{2} \times R_{\mathrm{BLR}}^{\mathrm{H} \beta} \simeq \sigma_{\mathrm{H} \alpha_{1}}^{2} \times R_{\mathrm{BLR}}^{\mathrm{H} \alpha_{1}} \simeq$ $\sigma_{\mathrm{H} \alpha_{2}}^{2} \times R_{\mathrm{BLR}}^{\mathrm{H} \alpha_{2}}$ is accepted (the $\mathrm{BH}$ masses estimated through the inner broad $\mathrm{H} \beta$ (i.e., observed broad $\mathrm{H} \beta$ ) should be similar to the mass estimated on the inner broad $\mathrm{H} \alpha$ ). Thus, the intermediate broad component of $\mathrm{H} \alpha$ for PG 0052 should lead to a different virial $\mathrm{BH}$ mass through the total observed broad $\mathrm{H} \beta$ (only one inner broad component in the observed spectrum) and the total observed broad $\mathrm{H} \alpha$ (one inner broad component plus one intermediate broad component), and to the result that PG 0052 should be one outlier in the plane of $\sigma_{\mathrm{H} \beta}^{2} / \sigma_{\mathrm{H} \alpha}^{2}$ versus $R_{\mathrm{BLR}}^{\mathrm{H} \alpha} / R_{\mathrm{BLR}}^{\mathrm{H} \beta}$.

Now let us check results of Equation (6) for PG $0052+251$. The flux ratio of the inner broad $\mathrm{H} \alpha$ to the intermediate broad $\mathrm{H} \alpha$ (the total observed broad $\mathrm{H} \alpha$ minus the intermediate broad $\mathrm{H} \alpha$ ) is about 2.5-3 (2.5 from the mean spectrum, and 3 for the observed spectra), the size ratio of the intermediate BLR (for intermediate broad $\mathrm{H} \alpha$ ) to the inner BLR (for the inner broad $\mathrm{H} \alpha$ ) is about 6-7. Then, the ratio of $\sigma_{\mathrm{H} \alpha}^{2} \times R_{\mathrm{BLR}}^{\mathrm{H} \alpha}$ to $\sigma_{\mathrm{H} \beta}^{2} \times R_{\mathrm{BLR}}^{\mathrm{H} \beta}$ should be about 2 based on Equation (6), which agrees well with the position of PG $0052+251$ in Figure 1. In Figure 1, the area marked by dotted lines shows the accepted range for the ratio of $\sigma_{\mathrm{H} \alpha}^{2} \times R_{\mathrm{BLR}}^{\mathrm{H} \alpha}$ to $\sigma_{\mathrm{H} \beta}^{2} \times R_{\mathrm{BLR}}^{\mathrm{H} \beta}$ under the size ratio of $R_{\mathrm{BLR}}^{\mathrm{H} \alpha_{2}} / R_{\mathrm{BLR}}^{\mathrm{H} \alpha_{1}} \sim 6-7$, based on Equation (6). Furthermore, it is simple to check and confirm the correlations shown in Equation (4). The results indicate that the outlier PG $0052+251$ in Figure 1 can be perfectly explained by properties of the intermediate broad component of $\mathrm{H} \alpha$, which further indicates that the intermediate broad component (or the intermediate BLR) is reasonable.

Before we end this subsection, we can compare our measured size of the intermediate BLR with reported results on the size of the intermediate BLR in the literature. Zhu et al. (2009) have found one correlation between the size of the intermediate BLR and central BH masses (Figure 7 in Zhu et al. 2009). If we accept $M_{\mathrm{BH}} \sim(3.69 \pm 0.76) \times 10^{8} M_{\odot}$ for PG 0052+251 (Peterson et al. 2004), then the estimated size of the intermediate BLR based on the correlation shown in Zhu et al. (2009) is consistent with our measured value of $\sim 10^{18} \mathrm{~cm}$, which provides further reliable evidence for the intermediate BLR.

\subsection{Extended Part of the Inner BLR?}

One question for the existence of the intermediate BLR is whether the component is the only probable extended part of the inner BLR, but not the one isolated region. We answer the question as follows. Based on the results above, there is a large distance between the inner and intermediate BLRs, about 600 light days. If the probable intermediate BLR is just the extended part of the inner BLR, the BLR of PG 0052 should have a much extended size. So, the large, extended size of the BLR should smooth the variations of the observed broad emission lines; in other words, there should be no apparent variations of broad emission lines. We check the effects of the extended size of the BLR on variations using the following mathematical procedure.

Before proceeding further, we accept the assumptions listed in Peterson (1993) for the reverberation-mapping technique: (1) continuum emission is from one central source, which is much smaller than the BLR, (2) both continuum emission and line emission are freely and isotopically propagating in central volume, (3) line emissions are in rapid response to ionizing continuum. Furthermore, we accept that line intensity from one region simply depends on the number density of the region, $I \propto N(r)$ (some detailed discussion for emission lines of AGNs can be found in Netzer 1990; Osterbroack \& Ferland 2006). Moreover, we simply accept that the BLR is a spherical shell (the center of the sphere is positioned on the central $\mathrm{BH}$ ) with a depth of about 600 light days (the inner layer radius is about 90 light days, the outer layer is about 700 light days). Then based on the geometrical structure, the response light curve of the emission line based on the input light curve of continuum emission can be created, as was done for 3C390.3 (Zhang 2011). The spherical shell (BLR) is first divided into $M$ layers. As long as $M$ is large enough, the effects of the depth of each layer can be totally ignored. The line intensity from each layer (with radius $r$ ) can be determined by

$$
I(r) \propto N(r) \propto 1 / r^{p} .
$$

In order to find more clear effects of the extended size of BLR, $p \sim 0$ is accepted. If $p$ was much larger, the outer part of the BLR would have few contributions to line emission. Thus, we select $p=0$; there is similar flux strength from clouds in 


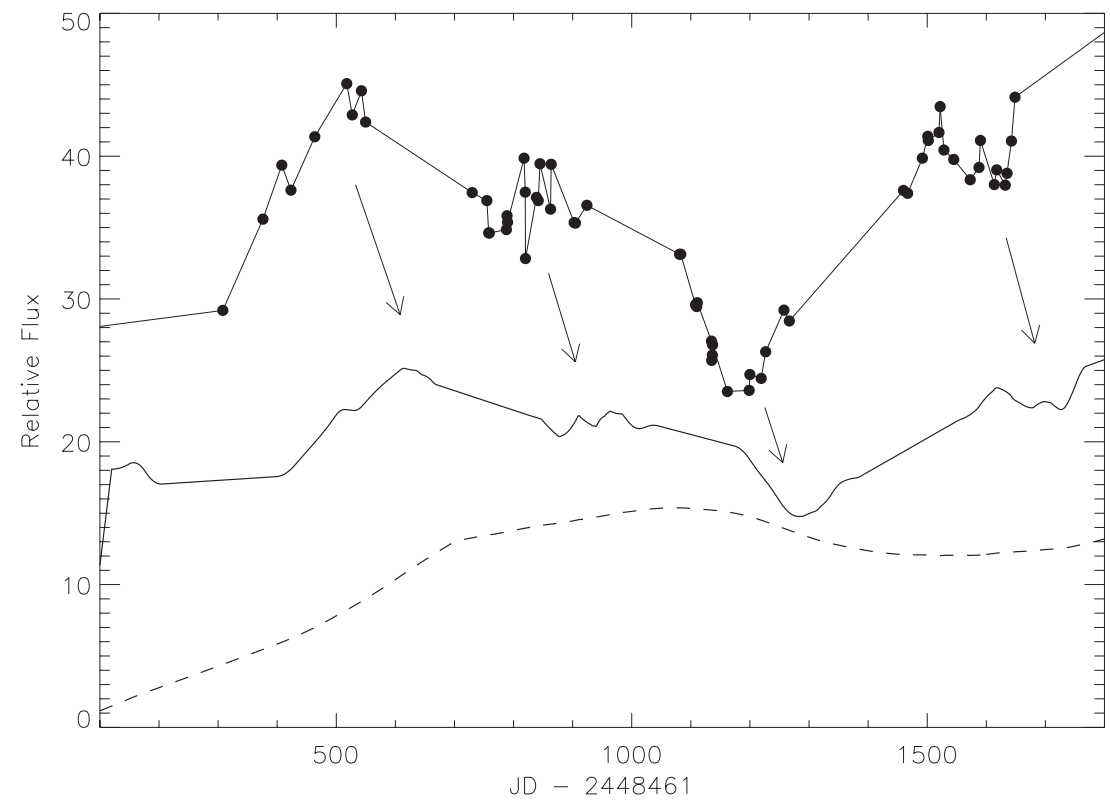

Figure 6. Effects of the extended size of the BLR on the observed light curve of the broad emission line. The solid line plus the solid circle represent the observed light curve of the continuum emission of PG 0052, the solid line represents the created light curve of the emission line for the BLR with an extended size of about 30 light days, and the dashed line represents the created light curve of the emission line for the BLR with an extended size of about 600 light days. The arrows show the positions of apparent features in the light curve of the continuum emission and in the corresponding created light curve of the emission line.

the inner part of the BLR and from clouds in the outer part of the BLR, which will show more clear effects of the extended size of the BLR on the observed light curve of the broad line. Once one layer meets the ionizing photos from the central source, the line intensity of the layer is changed immediately as

$$
I_{i}(t) \propto I_{i}(t-1) \times \frac{\operatorname{con}_{i}(t)}{\operatorname{con}_{i}(t-1)},
$$

where $t$ is the date, and $I_{i}$ and $c o n_{i}$ mean the line intensity from the $i$ th layer and the arriving continuum emission (a discrete data series with a time separation of 1 day) for the layer.

There is one point we should note in our mathematical procedure, which is that the simplest spherical shell geometry is assumed for the BLR. Actually, the geometry is perhaps different from the true structure of the BLR of PG 0052+251. As discussed in Gaskell (2009), Eracleous et al. (2009), Sluse et al. (2011), Bentz et al. (2010a), and references therein, the BLRs of AGNs have a flattened distribution and we always view them near pole-on, and BLR structures are very similar in most AGNs. Although the applied spherical shell geometry is oversimplified for PG $0052+251$, the results based on the simplified structure can still indicate the true effects of the extended size of the BLR on the observed light curve of the broad line. Different structures of the BLR should lead to different overall trends of observed light curves of broad lines (such as one example shown in Zhang 2011); the large extended size of the BLR still smooths the observed light curve of the broad line. As one example to demonstrate the effects of the extended size of the BLR, the oversimplified structure of the BLR is significantly valid.

Due to the simple procedure above, the response light curve of $\mathrm{H} \alpha$ based on the light curve of the continuum emission of PG 0052 can be created and shown in Figure 6. It is clear that the response light curve of $\mathrm{H} \alpha$ is very smooth if the extended size of the BLR is about 600 light days; i.e., if the calculated intermediate BLR of PG 0052 is just an extended part of the inner BLR. Furthermore, we also show one tested light curve of $\mathrm{H} \alpha$, if the BLR had a smaller extended size of about 30 light days. For the case with a small extended size of the BLR, some subtle features (the arrows in the figure) shown in the light curve of the continuum emission can be reflected in the light curve of the emission line; however, the features cannot be found in the created light curve of the emission line for the BLR with a larger extended size. In this subsection, to clearly describe the structures of the BLR of PG 0052 is not our objective. Thus, we do not show further results. The results shown in Figure 6 clearly indicate that the intermediate BLR cannot be treated as an extended part of the inner BLR for PG 0052, otherwise, the observed light curve of $\mathrm{H} \alpha$ would be much smoother.

\section{DISCUSSIONS}

After BLRs of AGNs have been studied for more than half a century, some information about the geometrical structures of the BLR has been mathematically determined by the transfer function in the reverberation-mapping technique through special mathematical methods (such as the maximum entropy method; Narayan \& Nityananda 1986; Peterson 1993; Peterson et al. 1994; Horne et al. 1991; Goad et al. 1993; Wanders \& Horne 1994; Pijpers \& Wanders 1994; Krolik 1994; Winge et al. 1995; Bentz et al. 2010a, 2010b; Sluse et al. 2011). Certainly, aside from the results obtained with mathematical methods, there are some statistical results about the structures of the BLRs of AGNs (some reviews can be found in Gaskell 2009; Eracleous et al. 2009; Down et al. 2010) based on the properties of observed broad emission lines, such as the proposed model of one much broader component for the wing of the broad emission line and one intermediate broad component for the core of the broad emission line (Zhu et al. 2009; Hu et al. 2008; Sulentic et al. 2000; Brotherton et al. 1994; Brotherton 1996; Mason et al. 1996; Bon et al. 2009; Popovic 2007). However, evidence is not sufficient enough to confirm the intermediate BLR of AGNs. In this paper, besides the fitted results for broad Balmer emission lines, properties of line cores from the PCA technique and the measured size of the expected intermediate BLR are further confirmed as evidence for the intermediate BLR of 
PG 0052+251. Besides one common inner component of the BLR (inner BLR) with the size of about 100 light days, there is one other seriously obscured intermediate component of the BLR (intermediate BLR) with the size of about 700 light days.

Before proceeding further, we first consider what geometry is envisioned for the intermediate BLR, which suffers much more reddening than the component giving rise to the inner BLR for PG $0052+251$. Actually, it is not difficult to answer this question. Obscuration for the intermediate BLR is not due to the dust torus but due to the high-density dust clouds (radially moving or not) between the line of sight and the intermediate BLR. The dust clouds have apparent effects on the intermediate BLR but no effects on the inner BLR due to the small size of the clouds. The dust clouds can be confirmed by a special kind of AGN, AGNs with their types changing between type 1 and type 2, such as Mrk1018 (Cohen et al. 1986; Goodrich 1990), NGC 7603 (Tohline \& Osterbrock 1976), NGC 2622, Mrk 609 (Goodrich 1990), etc. The large distance between the inner and intermediate BLRs for PG 0052 (about 600 light days) ensures enough space for the dust clouds.

Furthermore, we consider the virialization assumption (Gaskell 1988; Wandel et al. 1999; Peterson \& Wandel 1999) for the intermediate BLR. As discussed in Brotherton et al. (1994) through a simple photoionization model, properties of the inner and intermediate BLRs are consistent with the virialization assumption:

$$
V_{1}^{2} \times R_{\mathrm{BLR}, 1} \sim V_{2}^{2} \times R_{\mathrm{BLR}, 2}
$$

Here $R_{\mathrm{BLR}}$ means the size of the BLR (distance between the BLR and central $\mathrm{BH}$ ) and $V$ represents the rotating velocity of broad emission line clouds in the corresponding BLR (commonly, the line width of broad emission lines, the second moment). For PG $0052+251$, the sizes of the two components of the BLR and the line widths of the inner broad and intermediate broad components have been measured above,

$$
\left(\frac{\sigma_{\mathrm{H} \alpha_{1}}}{\sigma_{\mathrm{H} \alpha_{2}}}\right)^{2.0}=4.7 \pm 0.4 \simeq \frac{R_{\mathrm{BLR}}^{\mathrm{H} \alpha_{2}}}{R_{\mathrm{BLR}}^{\mathrm{H} \alpha_{1}}}=6 \pm 1.5 .
$$

Here, the mean value $\left(\sigma_{\mathrm{H} \alpha_{1}} / \sigma_{\mathrm{H} \alpha_{2}}\right)$ is calculated by the measured line parameters for all observed spectra of PG $0052+251$, which is consistent with the one from the mean spectrum of PG $0052+251$. The value of $R_{\mathrm{BLR}}^{\mathrm{H} \alpha_{2}} / R_{\mathrm{BLR}}^{\mathrm{H} \alpha_{1}}$ is calculated by the measured sizes of the BLR listed in Table 2. It is clear that the result is consistent with what we expect under the virialization method for the BLR.

Certainly, some effects on the measured line parameters of mapped AGNs should be discussed. As discussed in Kaspi et al. (2000), the variable Fe II lines could alter line parameter measurements. However, among the listed PG quasars in Table 1, PG 0052 and PG 0026 are the only two objects without apparent optical Fe II lines; the other five PG quasars have strong and apparent Fe II emission lines. However, only PG 0052 is an outlier in Figure 1. Thus, the effects of Fe II on measured line parameters can be totally ignored. Another question we should discuss is the size to which the BLR could change, such as in the simple discussion in Kaspi et al. (2000) and the detailed study and discussions for well-known mapped AGN NGC 5548 (Bentz et al. 2006, 2007; Denney et al. 2010; Peterson et al. 2004; Wanders $\&$ Peterson 1996). However, we can find a decreasing line width with the increasing size of the BLR, consistent with the expected results under the virialization assumption. Thus, the effects of the size of the BLR changing could not explain the outliers in Figure 1.

Before we end this section, we should note that it would be interesting to find candidates for AGNs with intermediate BLRs by the outliers in the plane of $R_{\mathrm{BLR}}^{\mathrm{H} \alpha} / R_{\mathrm{BLR}}^{\mathrm{H} \beta}$ versus $\sigma_{\mathrm{H} \beta}^{2} / \sigma_{\mathrm{H} \alpha}^{2}$, especially for objects with much larger values of $\left(\sigma_{\mathrm{H} \alpha}^{2} \times\right.$ $\left.R_{\mathrm{BLR}}^{\mathrm{H} \alpha}\right) /\left(\sigma_{\mathrm{H} \beta}^{2} \times R_{\mathrm{BLR}}^{\mathrm{H} \beta}\right)$. Thus, we expect that the mapped object NGC 4253 should be a strong candidate with an intermediate BLR.

Finally, a simple summary is as follows. We first check the properties of mapped AGNs collected from the literature in the plane of $\sigma_{\mathrm{H} \beta}^{2} / \sigma_{\mathrm{H} \alpha}^{2}$ versus $R_{\mathrm{BLR}}^{\mathrm{H} \alpha} / R_{\mathrm{BLR}}^{\mathrm{H} \beta}$. Commonly, virial $\mathrm{BH}$ masses based on the properties of observed broad $\mathrm{H} \alpha$ and $\mathrm{H} \beta$ should be a coincidence. However, among the mapped objects with measured sizes of BLR and line widths (second moment) based on long-period observed broad $\mathrm{H} \alpha$ and $\mathrm{H} \beta$, PG 0052 and NGC 4253 are two apparent outliers in the plane of $\sigma_{\mathrm{H} \beta}^{2} / \sigma_{\mathrm{H} \alpha}^{2}$ versus $R_{\mathrm{BLR}}^{\mathrm{H} \alpha} / R_{\mathrm{BLR}}^{\mathrm{H} \beta}$, which indicates that the BLRs of PG 0052 and NGC 4253 have some special characteristics. Then based on 55 public spectra of PG 0052 (Kaspi et al. 2000), the BLR of PG 0052 was studied in detail carefully. We find that the line width ratio of the total observed broad $\mathrm{H} \alpha$ to total observed broad $\mathrm{H} \beta$ is $\sim 0.7$, which is much smaller than the theoretical/observational value of $\sim 0.9$ found by Korista $\&$ Goad (2004) and Greene \& Ho (2005). Furthermore, the flux ratio of the total broad $\mathrm{H} \alpha$ to the total broad $\mathrm{H} \beta$ is about 6.8 (Balmer Decrement), which is not a reasonable value (mean value of 3.56 in Vanden Berk et al. 2001) for the blue quasar PG $0052+251$. Moreover, the properties of the line cores based on the PCA technique indicate there is one inner broad component and one seriously obscured intermediate broad component in the BLR. If the seriously obscured intermediate BLR was accepted, properties of PG 0052 in the plane of $\sigma_{\mathrm{H} \beta}^{2} / \sigma_{\mathrm{H} \alpha}^{2}$ versus $R_{\mathrm{BLR}}^{\mathrm{H} \alpha} / R_{\mathrm{BLR}}^{\mathrm{H} \beta}$ could be reproduced, which indicates that the intermediate BLR actually is appropriate for the mapped quasar PG 0052+251. Finally, the large distance between the inner and the intermediate components of the BLR based on CCF results (about 600 light days) indicates that the intermediate component is not an extended part of the inner component of the BLR.

We gratefully thank the anonymous referee for giving us constructive comments and suggestions to greatly improve our paper. Z.X.G. gratefully acknowledges the support from NSFC-11003034 and gratefully thanks Dr. Kaspi S. who provided us with the available observed spectra of PG 0052 (http://wise-obs.tau.ac.il/ shai/PG/), and gratefully thanks the AGNWATCH Project and Prof. Wang J.-M. for the discussions and constructive suggestions for the manuscript. This research has made use of NASA/IPAC Extragalactic Database (NED), which is operated by the Jet Propulsion Laboratory, California Institute of Technology, under contract with the National Aeronautics and Space Administration.

\section{APPENDIX}

\section{TO PROVE EQUATION (4)}

Based on the definition of the second moment (Peterson et al. 2004)

$$
\sigma^{2}=\frac{\int \lambda^{2} \times P_{\lambda} d \lambda}{\int P_{\lambda} d \lambda}-\left(\frac{\int \lambda P_{\lambda} d \lambda}{\int P_{\lambda} d \lambda}\right)^{2}=\frac{\int \lambda^{2} \times P_{\lambda} d \lambda}{\int P_{\lambda} d \lambda}-\lambda_{0}^{2},
$$




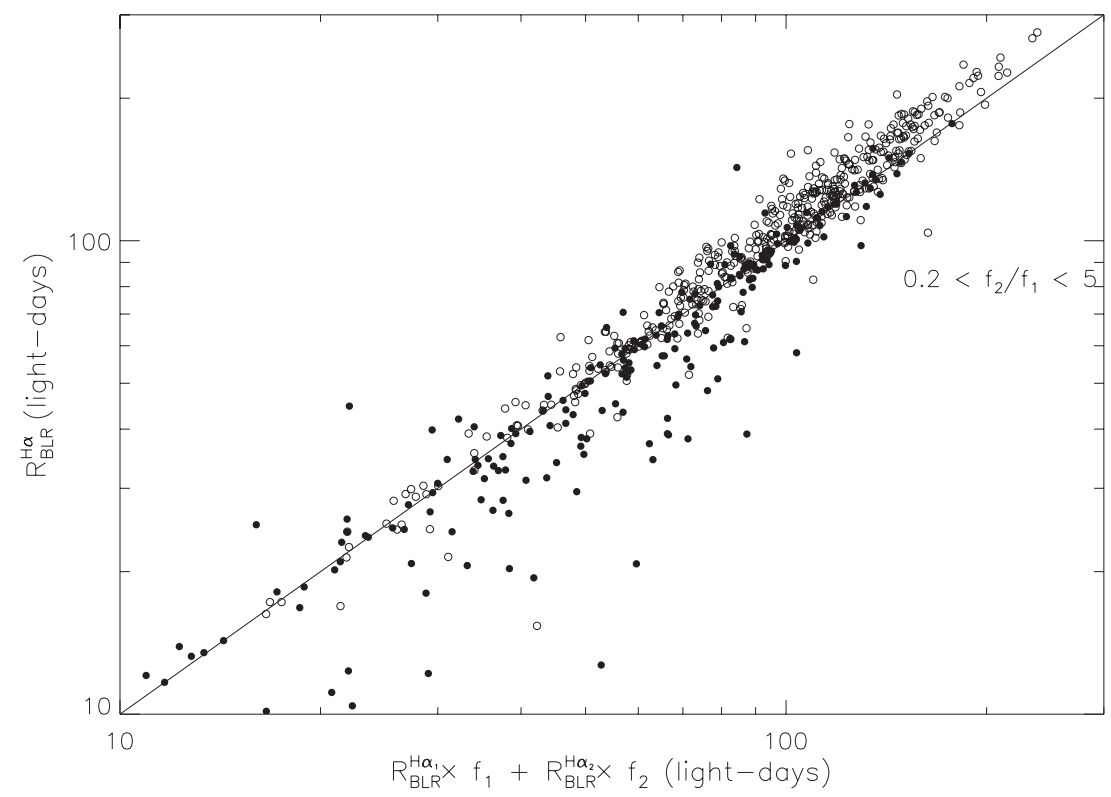

Figure 7. On the correlation between $R_{\mathrm{BLR}}^{\mathrm{H} \alpha}$ and $R_{\mathrm{BLR}}^{\mathrm{H} \alpha_{1}} \times f_{1}+R_{\mathrm{BLR}}^{\mathrm{H} \alpha_{2}} \times f_{2}$ for a total of 664 simulated data points. Open circles represent 444 data points with $f 2 / f 1 \geqslant 1$ and solid circles are for 220 data points with $f 2 / f 1 \leqslant 1$. The solid line represents the relation $R_{\mathrm{BLR}}^{\mathrm{H} \alpha}=R_{\mathrm{BLR}}^{\mathrm{H} \alpha_{1}} \times f_{1}+R_{\mathrm{BLR}}^{\mathrm{H} \alpha_{2}} \times f_{2}$.

where the function $P$ represents the line profile and $\lambda$ represents the wavelength. For broad $\mathrm{H} \alpha$ of PG 0052 which includes two components of $\mathrm{H} \alpha_{1}$ (the inner broad component) and $\mathrm{H} \alpha_{2}$ (the intermediate broad component), we can find the correlation between the line width of the total broad $\mathrm{H} \alpha\left(\sigma_{\mathrm{H} \alpha}\right)$ and line widths of the two components of $\mathrm{H} \alpha\left(\sigma_{\mathrm{H} \alpha_{1}}\right.$ and $\left.\sigma_{\mathrm{H} \alpha_{2}}\right)$ :

$$
\begin{aligned}
\sigma_{\mathrm{H} \alpha}^{2}= & f_{1} \times \sigma_{\mathrm{H} \alpha_{1}}^{2}+f_{2} \times \sigma_{\mathrm{H} \alpha_{2}}^{2}+f_{1} \times \lambda_{0}^{2}\left(\mathrm{H} \alpha_{1}\right) \\
& +f_{2} \times \lambda_{0}^{2}\left(\mathrm{H} \alpha_{2}\right)-\left[f_{1} \times \lambda_{0}\left(\mathrm{H} \alpha_{1}\right)+f_{2} \times \lambda_{0}\left(\mathrm{H} \alpha_{2}\right)\right]^{2} \\
= & f_{1} \times \sigma_{\mathrm{H} \alpha_{1}}^{2}+f_{2} \times \sigma_{\mathrm{H} \alpha_{2}}^{2}+G\left[\lambda_{0}\left(\mathrm{H} \alpha_{1}\right), \lambda_{0}\left(\mathrm{H} \alpha_{2}\right)\right], \quad(\mathrm{A} 2)
\end{aligned}
$$

where $f_{1}$ and $f_{2}$ are the flux ratios of the separated broad components of $\mathrm{H} \alpha$ to the total broad $\mathrm{H} \alpha$, i.e., $f_{1}=\int P_{\lambda, 1} d \lambda / \int P_{\lambda} d \lambda$, $f_{2}=\int P_{\lambda, 2} d \lambda / \int P_{\lambda} d \lambda$, and $f_{1}+f_{2}=1 . \lambda_{0}\left(\mathrm{H} \alpha_{1}\right)$ and $\lambda_{0}\left(\mathrm{H} \alpha_{2}\right)$ are the first moments (the center wavelengths) of the two components of the broad $\mathrm{H} \alpha$. It is clear that if the inner component and the intermediate broad component do not have largely different center wavelengths $\left(\lambda_{0}\left(\mathrm{H} \alpha_{1}\right) \simeq \lambda_{0}\left(\mathrm{H} \alpha_{2}\right)\right)$, the equation $\sigma_{\mathrm{H} \alpha}^{2} \simeq f_{1} \times \sigma_{\mathrm{H} \alpha_{1}}^{2}+f_{2} \times \sigma_{\mathrm{H} \alpha_{2}}^{2}$ can be safely accepted.

To mathematically prove equation $R_{\mathrm{BLR}}^{\mathrm{H} \alpha} \simeq f_{1} \times$ $R_{\mathrm{BLR}}^{\mathrm{H} \alpha_{1}}+f_{2} \times R_{\mathrm{BLR}}^{\mathrm{H} \alpha_{2}}$ is very difficult. Here, we prove the equation by following the Monte Carlo method, based completely on homogeneous light curves of continuum emission $(C(t))$ and broad line emission $(L(t))$ of the well-known reverberation-mapped AGN NGC 5548 (Peterson et al. 2002) collected from the AGNWATCH project (http://www.astronomy.ohio-state.edu/ agnwatch/). Light curves of inner and intermediate broad $\mathrm{H} \alpha$ are created through the bootstrap method (the commonly used Monte Carlo method creates a mock population from a given sample of data) applied to the light curve of the broad line $\mathrm{H} \beta$ of NGC 5548. The observed light curve of NGC 5548, including $N$ data points, can be described as $\left[t_{i}\right.$, flux $\left._{i}\right]$, where the index $i=1,2, \ldots, N$ means the $i$ th data points in the light curve, and $t$ and flux represent the observational date and corresponding flux density of the emission line. In order to simplify our mathematical procedure, the dates $t_{i}$ are recreated with isolated integral values with a step of 1 day based on the observed data series of NGC 5548. Then, mock light curves of the inner and intermediate broad components of $\mathrm{H} \alpha$ can be created with the following two steps. On the first step, using the bootstrap method, a new sample with an index from 1 to $N, k=1, \ldots N$, is created. Certainly, there are some values that will be the same in the sample of $k$. On the second step, the mock light curve of the inner broad $\mathrm{H} \alpha$ is created by $\left[t_{i}\right.$, flux $\left._{k}\right]$, the mock light curve of the intermediate broad $\mathrm{H} \alpha$ is created by $\left[t_{i}+\Delta\right.$, flux $\left.x_{k} \times f_{\text {sca }}\right]$, where $\Delta \in[2,160]$ days are integral values and represent distances between the inner BLR and intermediate BLR, and $f_{\text {sca }} \in[0.2,5]$ represents the flux density ratio of the intermediate broad $\mathrm{H} \alpha$ to the inner broad $\mathrm{H} \alpha$. The values of $f_{\text {sca }}$ ensure that both of the broad components of $\mathrm{H} \alpha$ are apparent. Parameter $\Delta$ ensures that there is a longer distance between the intermediate BLR and central $\mathrm{BH}$ than the distance between the inner BLR and central BH. Then, the two steps above are repeated, until there are enough mock light curves. Furthermore, based on the bootstrap method, the effects of different geometrical structures of the inner and intermediate components of the BLR have been simply included (simple results of the effects of the geometric structures of the BLR on the measured size of the BLR can be found in Zhu et al. 2009). Based on the created mock light curves (date and flux density: $\left[t_{i, \text { inner }}\right.$, flux $\left._{k, \text { inner }}\right]$ and $\left.\left[t_{i, \text { inter }}, f l u x_{k, \text { inter }}\right]\right)$ of the inner and intermediate components, it is easy to create the mock light curves $\left(\left[t_{i, \text { tot }}, f l u x_{k, \text { tot }}\right]\right)$ of the total broad line (the inner component plus the intermediate component) by

$$
\begin{aligned}
& t_{i, \text { tot }}=t_{i, \text { inter }} \text { if } t_{i, \text { inter }}=t_{i, \text { inner }} \\
& t_{i, \text { tot }} \text { rejected if } t_{i, \text { inter }} \neq t_{i, \text { inner }} \\
& \text { flux }_{k, \text { tot }}=\text { flux }_{k, \text { inner }}+\text { flux }_{k, \text { tot }} \text { if } t_{i, \text { inter }}=t_{i, \text { inner }} \\
& \text { flux }_{k, \text { tot }} \text { rejected if } t_{i, \text { inter }} \neq t_{i, \text { inner }} .
\end{aligned}
$$

Then, the values of $R_{\mathrm{BLR}}^{\mathrm{H} \alpha}, R_{\mathrm{BLR}}^{\mathrm{H} \alpha_{1}}$, and $R_{\mathrm{BLR}}^{\mathrm{H} \alpha_{2}}$ can be determined by $\mathrm{CCF}$. Here we notice that only the CCF result with one apparent peak is accepted. Figure 7 shows the correlation (coefficient 0.97 with significant deviation from zero $P_{\text {null }} \sim 0$ for 664 simulated data points) between $R_{\mathrm{BLR}}^{\mathrm{H} \alpha}$ and $R_{\mathrm{BLR}}^{\mathrm{H} \alpha_{1}} \times f_{1}+R_{\mathrm{BLR}}^{\mathrm{H} \alpha_{2}} \times f_{2}$ $\left(f_{1}=1 /\left(1+f_{\text {sca }}\right)\right.$ and $\left.f_{2}=f_{\text {sca }} /\left(1+f_{\text {sca }}\right)\right)$. It is clear that the 
expected relation about the size of the BLR in Equation (4) can be commonly accepted. Furthermore, the results shown in Figure 7 even indicate that there are two apparent components in the light curve of the broad line; the CCF could be singlepeaked, not double-peaked, in appearance.

\section{REFERENCES}

Alexander, T. 1997, in Astronomical Time Series, ed. D. Maoz, A. Sternberg, \& E. M. Leibowitz (Dordrecht: Kluwer), 163

Assef, R. J., Denney, K. D., Kochanek, C. S., et al. 2010, ApJ, in press (arXiv:1009.1145)

Barth, A. J., Nguyen, M. L., Malkan, M. A., et al. 2011, ApJ, 732, 121

Bentz, M. C., Denney, K. D., Cackett, E. M., et al. 2007, ApJ, 662, 205

Bentz, M. C., Horne, K., Barth, A. J., et al. 2010a, ApJ, 720, L46

Bentz, M. C., Peterson, B. M., Pogge, R. W., Vestergaard, M., \& Onken, C. A. 2006, ApJ, 644, 133

Bentz, M. C., Walsh, J. L., Barth, A. J., et al. 2009, ApJ, 705, 199

Bentz, M. C., Walsh, J. L., Barth, A. J., et al. 2010b, ApJ, 716, 993

Blandford, R. D., \& Mckee, C. F. 1982, ApJ, 255, 419

Bon, E., Popovic, L. C., Gavrilovic, N., Mura, G. L., \& Mediavilla, E. 2009, MNRAS, 400, 924

Brotherton, M. S. 1996, ApJS, 102, 1

Brotherton, M. S., Wills, B. J., Francis, P. J., \& Steidel, C. C. 1994, ApJ, 430 , 495

Cohen, R. D., Rudy, R. J., Puetter, R. C., Ake, T. B., \& Foltz, C. B. 1986, ApJ, 311,135

Collin, S., Kawaguchi, T., Peterson, B. M., \& Vestergaard, M. 2006, A\&A, 456, 75

Denney, K. D., Peterson, B. M., Dietrich, M., Vestergaard, M., \& Bentz, M. C. 2009a, ApJ, 692, 246

Denney, K. D., Peterson, B. M., Pogge, R. W., et al. 2009b, ApJ, 704, L80

Denney, K. D., Peterson, B. M., Pogge, R. W., et al. 2010, ApJ, 721, 715

Dimitrijevic, M. S., Popovic, L. C., Kovacevic, J., Dacic, M., \& Illic, D. 2007, MNRAS, 374, 1181

Down, E. J., Rawlings, S., Sivia, D. S., \& Baker, J. C. 2010, MNRAS, 401, 633

Edelson, R. A., \& Krolik, J. H. 1988, ApJ, 333, 646

Eracleous, M., Lewis, K. T., \& Flohic, H. M. L. G. 2009, New Astron. Rev., 53, 133

Francis, P. J., Hewett, P. C., Foltz, C. B., \& Chaffee, F. H. 1992, ApJ, 398, 476

Gaskell, C. M. 1988, ApJ, 325, 114

Gaskell, C. M. 2009, New Astron. Rev., 53, 140

Gaskell, C. M. 2010, ApJ, submitted (arXiv:1008.1057)

Gaskell, C. M., \& Peterson, B. 1987, ApJS, 65, 1

Gaskell, C. M., \& Sparke, L. S. 1986, ApJ, 305, 175

Goad, M. R., O'Brien, P. T., \& Gondhalekar, P. M. 1993, MNRAS, 263, 149

Goodrich, R. W. 1990, ApJ, 355, 88

Greene, J. E., \& Ho, L. C. 2005, ApJ, 630, 122

Greene, J. E., Hood, C. E., Barth, A. J., et al. 2010, ApJ, 723, 409

Horne, K., Welsh, W. F., \& Peterson, B. M. 1991, ApJ, 367, L5

Hu, C., Wang, J.-M., Ho, L. C., et al. 2008, ApJ, 683, L115

Kaspi, S., Maoz, D., Netzer, H., et al. 2005, ApJ, 629, 61

Kaspi, S., Smith, P. S., Netzer, H., et al. 2000, ApJ, 533, 631
Kelly, B. C., \& Bechtold, J. 2007, ApJS, 168, 1

Korista, K. T., \& Goad, M. R. 2004, ApJ, 606, 749

Krause, M., Burkert, A., \& Schartmann, M. 2011, MNRAS, 411, 550

Krolik, J. H. 1994, IAU Symp. 159, Multi-Wavelength Continuum Emission of AGN, ed. T. L.-J. Courvoisier \& A. Blecha (Cambridge: Cambridge Univ. Press), 163

Mason, K. O., Puchnarewicz, E. M., \& Jones, L. R. 1996, MNRAS, 283, L26

McLure, R. J., \& Dunlop, J. S. 2004, MNRAS, 352, 1390

Narayan, R., \& Nityananda, R. 1986, ARA\&A, 24, 127

Netzer, H. 1990, in Active Galactic Nuclei, ed. R. D. Blandford, H. Netzer, \& L. Woltjer (Berlin: Springer), 137

Netzer, H., \& Marziani, P. 2010, ApJ, 724, 318

Onken, C. A., Ferrarese, L., Merritt, D., et al. 2004, ApJ, 615, 645

Osterbroack, D. E., \& Ferland, G. J. 2006, Astrophysics of Gaseous Nebulae and Active Galactic Nuclei (2nd ed.; Mill Valley, CA: Univ. Science Books)

Osterbrock, D. E., \& Mathews, W. G. 1986, ARA\&A, 24, 171

Pancoast, A., Brewer, B. J., \& Treu, T. 2011, ApJ, 730, 139

Peterson, B. M. 1993, PASP, 105, 247

Peterson, B. M. 2010, in IAU Symp. 267, Co-Evolution of Central Black Holes and Galaxies, ed. B. M. Peterson, R. S. Somerville, \& T. Storchi-Bergmann (Cambridge: Cambridge Univ. Press), 151

Peterson, B. M., Alloin, D., Axon, D., et al. 1992, ApJ, 392, 470

Peterson, B. M., Balonek, T. J., Barker, E. S., et al. 1991, ApJ, 368, 119

Peterson, B. M., \& Bentz, M. C. 2006, New Astron. Rev., 50, 796

Peterson, B. M., Berlind, P., Bertram, R., et al. 1994, ApJ, 425, 622

Peterson, B. M., Berlind, P., Bertram, R., et al. 2002, ApJ, 581, 197

Peterson, B. M., Ferrarese, L., Gilbert, K. M., et al. 2004, ApJ, 613, 682

Peterson, B. M., \& Wandel, A. 1999, ApJ, 521, L95

Peterson, B. M., Wanders, I., Horne, K., \& Collier, S. 1998, PASP, 110, 660

Pijpers, F. P., \& Wanders, I. 1994, MNRAS, 271, 183

Popovic, L. C. 2007, in ESO Astrophys. Symp., European Southern Observatory Series, Exploring the Cosmic Frontier: Astrophysical Instruments for the 21st Century, ed. A. P. Lobanov et al. (series editor B. Leibundgut; Berlin: Springer), 191

Press, W. H., Teukolsky, S. A., Vetterling, W. T., \& Flannery, B. P. 1992, Numerical Recipes in Fortran 77 (2nd ed.; Cambridge: Cambridge Univ. Press), 340

Rafiee, A., \& Hall, P. B. 2011, ApJS, 194, 42

Shen, J., Vanden Berk, D. E., Schneider, D. P., \& Hall, P. B. 2008, AJ, 135, 928

Sluse, D., Schmidt, R., Courbin, F., et al. 2011, A\&A, 528, 100

Sulentic, J. W., Bachev, R., Marziani, P., Negrete, C. A., \& Dultzin, D. 2007, ApJ, 666,757

Sulentic, J. W., Marziani, P., \& Dultzin-Hacyan, D. 2000, ARA\&A, 38, 521

Sulentic, J. W., Repetto, P., Stirpe, G. M., et al. 2006, A\&A, 456, 929

Tohline, J. E., \& Osterbrock, D. E 1976, ApJ, 210, L117

Vanden Berk, D. E., Richards, G. T., Bauer, A., et al. 2001, AJ, 122, 549

van Groningen, E., \& Wanders, I. 1992, PASP, 104, 700

Vestergaard, M. 2002, ApJ, 571, 733

Wandel, A., Peterson, B. M., \& Malkan, M. A. 1999, ApJ, 526, 579

Wanders, I., \& Horne, K. 1994, A\&A, 289, 7

Wanders, I., \& Peterson, B. M. 1996, ApJ, 466, 174

Wang, T.-G., \& Zhang, X.-G. 2003, MNRAS, 340, 793

White, R. J., \& Peterson, B. M. 1994, PASP, 106, 876

Winge, C., Peterson, B. M., Horne, K., et al. 1995, ApJ, 445, 680

Zhang, X.-G. 2011, MNRAS, accepted (arXiv:1107.0455)

Zhu, L., Zhang, S.-N., \& Tang, S. 2009, ApJ, 700, 1173 